DIVISION OF THE HUMANITIES AND SOCIAL SCIENCES

CALIFORNIA INSTITUTE OF TECHNOLOGY

PASADENA, CALIFORNIA 91125

STRATEGIC VOTING IN SEQUENTIAL COMMITTEES

Matias laryczower

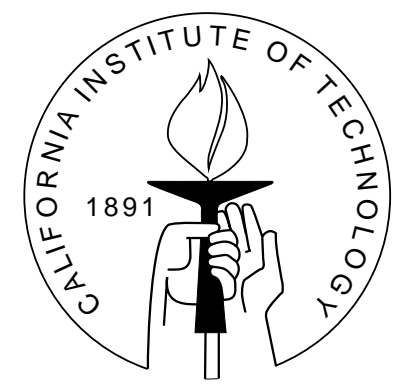

SOCIAL SCIENCE WORKING PAPER 1275

August 2007 


\title{
Strategic Voting in Sequential Committees
}

\author{
Matias Iaryczower
}

\begin{abstract}
We consider strategic voting with incomplete information and partially common values in sequential committees. A proposal is considered against the status quo in one committee, and only upon its approval advances for consideration in a second committee. Committee members (i) are privately and imperfectly informed about an unobservable state of nature which is relevant to their payoffs, and (ii) have a publicly observable bias with which they evaluate information. We show that the tally of votes in the originating committee can aggregate and transmit relevant information for members of the second committee in equilibrium, provide conditions for the composition and size of committees under which this occurs, and characterize all three classes of voting equilibria with relevant informative voting.
\end{abstract}

JEL classification numbers: D72, D78, C72

Key words: strategic voting, committees, information aggregation, sequential voting, bicameral legislatures. 


\title{
Strategic Voting in Sequential Committees*
}

\author{
Matias Iaryczower ${ }^{\dagger}$
}

\section{Introduction}

Voting of bills in bicameral legislatures has a sequential structure: a bill is originated in one chamber, and passes to the other chamber for consideration only after having been voted by a (possibly qualified) majority of representatives on the floor. This sequential arrangement of committees is in no way unique to bicameral legislatures. Still in the legislative arena, bills are typically considered by the floor of legislative bodies only after being approved by a majority of votes in the relevant standing committee. And in universities, faculty appointments typically require the approval of an "administrative" committee following the approval of a committee composed of faculty members of the relevant department.

A stylized fact common to all these examples is the outcome of the vote in the first committee can influence the outcome of the vote in the second committee beyond the binary decision of whether to approve or reject the alternative in the first committee: the larger the tally of votes in favor of the proposal in the initiating committee, the highest its success rate in the receiving committee. ${ }^{1}$ In this paper, we propose a simple explanation for this stylized fact. If committee members have private information about the relative value of the alternatives under consideration, voting outcomes can aggregate and transmit relevant information to members of the receiving committee. What is less straightforward is whether members of the originating committee will have incentives to vote informatively in equilibrium, and if so under what conditions. Which compositions and sizes of committees facilitate or hinder the transmission and aggregation of information in this environment?

${ }^{*}$ I am thankful to to Juliana Bambaci, Randy Calvert, Federico Echenique, Navin Kartik, Santiago Oliveros, and participants at the MPSA annual conference for comments to earlier versions of this paper. All errors remain my own.

${ }^{\dagger}$ Division of Humanities and Social Sciences, California Institute of Technology, Pasadena, California 91125, USA, email: miaryc@hss.caltech.edu

${ }^{1}$ As Oleszek (2004) notes regarding committees in the U.S. House of Representatives, "Bills voted out of committee unanimously stand a good chance on the floor. A sharply divided committee vote presages an equally sharp dispute on the floor (pg. 102)" 
To assess these questions, we develop a simple model of strategic voting with incomplete information and partially common values in sequential committees. The model builds on the seminal contributions of Austen-Smith and Banks (1996), and Feddersen and Pesendorfer $(1997,1998)$. As usual in the literature, committee members are privately and imperfectly informed about an unobservable state of nature which is relevant to their payoffs. Here, however, voting does not occur in single-committee systems. Instead, a proposal can prevail only by defeating the status quo by (possibly qualified) majority voting first in one and, provided it is successful there, then in a second committee (whose members, we assume, can observe the vote outcome in the initiating committee).

What does and what does not change vis-a-vis the standard single-committee setting? Note first that the strategic problem of members of the receiving committee is essentially the same as that of members of a single committee: in deciding their vote, individuals of the receiving committee care only about the event in which they are pivotal, and they are pivotal in the traditional sense of being the decisive vote in a divided committee (the standard-pivotal motive). The possibility of observing the outcome of the vote in the originating committee, however, introduces two main differences in the incentives of members of both committees. First, members of the receiving committee can condition their behavior on the realization of votes in the originating committee. When some members of the originating committee vote informatively, the tally of the votes in favor of the alternative becomes an informative public signal for members of the receiving committee, allowing different voting strategies to be equilibria in the second committee for different voting outcomes in the first committee. Second, as opposed to members of the receiving committee, members of the originating committee can influence the outcome both in the traditional sense of killing or passing the proposal in their committee, and by influencing the beliefs of members of the receiving committee regarding the relative value of the two alternatives (what we call the signal-pivotal voting motive).

We show that there are two classes of voting equilibria in which the tally of votes in favor of the proposal in the originating committee transmits relevant information to members of the receiving committee. In the first class, the receiving committee unconditionally (independently of the private information of its members) kills the proposal following sufficiently low vote tallies in the originating committee, and unconditionally approves the proposal otherwise. In equilibria of this class, informative voting occurs only in the originating committee; the second committee acts only to raise the hurdle that the alternative has to surpass in the first committee to defeat the status quo in equilibrium. As a result, the strategic problem of members of the originating committee in equilibria of this class resembles that of members of a single committee: their vote decision is guided by the standard-pivotal voting motive, as amended by the endogenous majority rule implied by the equilibrium behavior of members of the receiving committee. We call equilibria of class endogenous majority rule (EMR) voting equilibria.

The second class encompasses voting equilibria in which not only members of the originating committee vote informatively, but so do - following some realizations of the vote in the originating committee - members of the receiving committee. In particular, we show 
that in any equilibrium of this class there is a responsive set of initiating-committee voting outcomes in which the probability of the proposal being accepted increases (strictly) with the tally of votes in favor of the proposal in the originating committee. This occurs either because the number of individuals voting informatively in the receiving committee increases with higher tallies as a result of individuals switching from voting against the proposal unconditionally to voting informatively, or because the number of informative votes decreases with higher tallies as a result of individuals switching from voting informatively to voting for the proposal unconditionally, or both. As a result, in voting equilibria with relevant two-sided informative voting, the voting behavior of members of the originating committee is guided by a signal-pivotal motive.

Voting equilibria with transmission of information between committees have to be of one of the classes above. But under what conditions, if any, do EMR and two-sided informative voting equilibria exist? What in particular are the implications for the size and composition of committees? We address these questions in a setting that allows for open conflicts of interests between committee members: individuals are biased for or against the status quo, and this bias is public information. The distinction boils down to a different threshold with which individuals of different types evaluate information: conservatives - those biased for the status quo - require overwhelming evidence in favor of the proposal to prefer it over the status quo, and similarly liberals require overwhelming evidence against the proposal to favor the status quo. ${ }^{2}$ To make this distinction meaningful, we assume that an individual's own private information can never overturn the preference between alternatives implied by the bias.

In this setting, we establish existence of EMR and two-sided informative voting equilibria for plausible conditions on the size and composition of committees. We show that a key determinant for existence of equilibria of these classes is the "partisan" (ideological) composition of the receiving committee, and specifically whether conservatives can or can not block the passage of the proposal in the receiving committee. When they can, there is always an EMR voting equilibrium with $k$ conservatives in the originating committee voting informatively as long as the total number of conservatives in the originating committee is sufficiently large. When instead liberals are a winning coalition in the receiving committee, an equilibrium of this class can only exist if liberals are a winning coalition in both committees. Moreover, when this exists, the number of informative votes is bounded above by the majority premium of liberals in the originating committee.

Endogenous Majority Rule voting equilibria have attractive properties - they are extremely simple and also robust to sequential voting within each committee - but they are also inefficient, as no information from members of the receiving committee is incorporated in the collective decision. Third, then, we show that under some conditions the relevant majority can do better than in the most informative EMR voting equilibrium by simply delegating all relevant decision making to the receiving committee. For simple majority rule, in particular, the condition boils down to a comparison between the

\footnotetext{
${ }^{2}$ This is essentially without loss of generality, as we can capture the common interest case allowing for one type only.
} 
majority premium of liberals in each committee when liberals are a winning coalition in the receiving committee, but between the majority premium of conservatives in the receiving committee and the total number of conservatives in the originating committee when instead conservatives are a blocking coalition in the receiving committee.

Finally, we address existence of voting equilibria with two-sided relevant informative voting. We show that for an equilibrium of this class to exist it is sufficient that conservatives form a blocking coalition in the receiving committee and that the number of conservatives in the originating committee is sufficiently large. We also show, however, that there can exist a voting equilibrium with relevant two sided informative voting in which each of a small number of conservatives in the originating committee votes informatively. Moreover, this strategy profile remains an equilibrium when voting within each committee is allowed to be sequential as well. Finally, we show that when liberals are a winning coalition in the receiving committee it is both necessary and sufficient that liberals also control the originating committee for a voting equilibrium with relevant two sided informative voting that is robust to sequential voting within each committee to exist. Thus also in this class it is key whether conservatives can or cannot block the passage of the proposal in the receiving committee.

\section{Relation with the Literature}

This paper builds on the pioneering contributions of Austen-Smith and Banks (1996) and Feddersen and Pesendorfer $(1997,1998)$, and connects at least three strands of related research.

First, Piketty (2000) and Razin (2003) also build around the idea of voting as communicating, where voters have some type of signal-pivotal voting motive. In Piketty (2000), however, there are two outcomes corresponding to two stages of choices (an electorate chooses by plurality rule between two alternatives, the winner is implemented for one period, and then competes against a third alternative to be implemented in a second period), and the focus is on the inefficiency caused on the intermediate choice by the desire of voters to communicate information relevant to the second choice. In our sequential committees, this intermediate stage of payoffs simply does not exist, as the alternative approved by the first committee does not become an outcome - and hence does not influence payoffs - until also approved by the second committee. In Razin (2003), on the other hand, there is only one stage of voting, but the elected candidate uses the outcome of the vote to select the policy she will implement, in a single dimensional policy space. This richer space allows the outcome to be strictly responsive to the tally of votes for the winner in the election. In our setting, instead, this responsiveness comes with the probability of the proposal being passed in the receiving committee being increasing in the tally in the first committee. ${ }^{3}$

\footnotetext{
${ }^{3}$ In both of these papers, a first stage of voting communicates information for a second stage relevant to the determination of policy. A similar phenomenon arises in this regard when players can explicitly
} 
Our paper also relates to several papers exploring an alternative kind of sequentiality in voting in committees. In Dekel and Piccione (2000), as in Fey (1998), Wit (1997), Battaglini (2005), Battaglini, Morton, and Palfrey (2007), Callender (2007), and Ali and Kartik (2006), the focus is on sequential voting among members of a single committee: individuals can vote after observing prior votes by other voters in the population, but all votes are then aggregated in the same tally and the collective choice is determined by majority rule. None of these papers, however, considers sequential voting between committees. The two approaches provide complementary lessons for the study of hybrid systems lying in between these models, as that employed in the US presidential primaries.

Third is the also very closely related paper by Maug and Yilmaz (2002), which studies simultaneous voting in two committees in a setting similar to the one considered here (committees, however, are internally homogeneous, divided by type of voter). While the two papers are clearly complementary, simultaneous voting among committees leads to very different voting incentives to those faced by individuals in our setting, as there is no role for signaling to members of the receiving committee, and no way to condition behavior on history of play. ${ }^{4}$

Finally, this paper contributes to the literature on transmission of information from (standing) committees to the whole assembly pioneered by Gilligan and Krehbiel (1987). Gilligan and Krehbiel build - as do to our knowledge all subsequent contributions in the literature - on the seminal contribution of Crawford and Sobel (1982), and as a result treat both the committee and the floor (our originating and receiving committees) as unitary actors with the preferences of the respective median voters. Our analysis suggests that this assumption can be quite problematic. We do not handle here however information acquisition. This is a natural (and interesting) extension of the model that we leave for future research.

\section{The Model}

A group of individuals arranged in two committees, $C_{0}$ and $C_{1}$, choose between a proposal $A$ and a status quo $Q$, both lying in an arbitrary policy space $X$. Committee $j$ is populated by an odd number $n_{j}$ of individuals, and the collective choice of each committee $j$ is determined by voting under a $R_{j}$-majority rule without abstention. Formally, letting $v_{i} \in\{-1,1\}$ denote $i$ 's vote against $(-1)$ or in favor (1) of the proposal, $t_{j}\left(v_{j}\right) \equiv \sum_{i \in C_{j}} v_{i}$ the net tally of votes in favor of the proposal in $C_{j}$, and $z_{j} \in\{Q, A\}$ the policy choice

deliberate prior to a voting stage. For models of voting with deliberation, see Coughlan (2000), AustenSmith and Feddersen (2005), Austen-Smith and Feddersen (2006), and Gerardi and Yariv (2007). We return to the possibility of deliberation in the discussion.

${ }^{4}$ The emphasis in Maug and Yilmaz (2002) is on the efficiency comparison of unicameral and (simultaneous) bicameral systems, and not on the positive or behavioral properties. We address this in Iaryczower (2007). The comparison of unicameralism and bicameralism calls a much broader literature to the one we can review here (but see Tsebelis and Money (1997), Cutrone and McCarty (2005), and references within). 
in $C_{j}, z_{j}=A$ if and only if $t_{j}\left(v_{j}\right) \geq r_{j}$, for an odd integer $r_{j}$ such that $1 \leq r_{j} \leq n_{j}$ (thus $R_{j}=\frac{n_{j}+r_{j}}{2}$ ). Voting is simultaneous in each committee, but sequential between committees. In particular, we assume the following simple institutional environment: the alternatives are first voted on in the originating committee $C_{0}$. If the proposal defeats the status quo in the originating committee $\left(t_{0}\left(v_{0}\right) \geq r_{0}\right)$, the alternatives are then voted on in the receiving committee $C_{1}$, where $z_{1}=A$ if and only if $t_{1}\left(v_{1}\right) \geq r_{1}$. If $t_{j}\left(v_{j}\right)<r_{j}$ in any chamber $j$, the status quo remains $(z=Q)$.

There are two equally likely realizations of an unobservable state of the world, $\omega \in$ $\left\{\omega_{Q}, \omega_{A}\right\}$, and each individual $i \in C_{j}$ receives a private, imperfectly informative signal $s_{i} \in\{-1,1\}$, distributed independently conditional on the state, such that $\operatorname{Pr}\left(s_{i}=1 \mid \omega=\right.$ $\left.\omega_{A}\right)=\operatorname{Pr}\left(s_{i}=-1 \mid \omega=\omega_{Q}\right)=q>1 / 2$ (the restriction to uninformative priors is without loss of generality). Individuals' preferences have an ideological and a common value component. Each individual $i \in C_{j}$ has a publicly known ideology bias $b_{i}$ either for or against the proposal, and we say that $i$ is either a liberal or a conservative, respectively. Liberals and conservatives differ in their ranking of alternatives conditional on observing the same information. In particular, (moderate) liberals prefer the proposal to the status quo whenever $\operatorname{Pr}(\omega=A \mid \mathcal{I})>\pi_{A}$ for some $\pi_{A}<1 / 2$, while (moderate) conservatives prefer the proposal to the status quo whenever $\operatorname{Pr}(\omega=A \mid \mathcal{I})>\pi_{Q}$ for some $\pi_{Q}>$ $1 / 2 .{ }^{5}$ We will assume throughout, moreover, that biases are sufficiently strong relative to the precision of the private information so that for any individual $i$, knowledge of the realization of her own signal $s_{i}$ would not be enough to revert her desired policy outcome; i.e., we will assume that $\pi_{Q}>q$ and $\pi_{A}<1-q$.

A $b$-biased individual can be one of two possible types. With probability $1-\alpha, i$ is a moderate, and has the preferences described above. With probability $\alpha>0$, individual $i$ is a partisan and always votes her bias. We will focus on equilibria of the game as $\alpha \rightarrow 0$, and restrict attention to pure (symmetric or asymmetric) anonymous strategy profiles. ${ }^{6}$ A pure strategy for a (moderate) individual $i \in C_{j}$ is a mapping $\sigma_{i}$ from the set of signals $\{-1,1\}$ and feasible histories $H^{j}$ to a vote $v_{i} \in\{-1,1\}$. ${ }^{7}$ Since $h^{0}=\emptyset$, we

\footnotetext{
${ }^{5}$ To be more precise, we assume that for a given $\lambda_{i} \in(0,1 / 2), i \in C_{j}$ obtains payoff $\lambda_{i}$ if the policy outcome matches his or her bias (if $\left.z=b_{i}\right)$, and payoff $\left(1-\lambda_{i}\right)$ if the policy outcome matches the state (if $z=\omega$ ). Letting $f(z \mid \omega)=1$ if $z=\omega, 0$ otherwise, and $W\left(z ; b_{i}\right)=1$ if $z=b_{i}, 0$ otherwise, we write the payoff of individual $i$ with bias $b$ given policy outcome $z$ as: $U(z ; b)=\lambda_{i} W(z ; b)+\left(1-\lambda_{i}\right) f(z \mid \omega)$. Thus, given information $\mathcal{I}, i$ has induced preferences $E[U(z ; b) \mid \mathcal{I}]=\lambda_{i} W(z ; b)+\left(1-\lambda_{i}\right) \operatorname{Pr}(\omega=z \mid \mathcal{I})$, and $E[U(A ; b) \mid \mathcal{I}]>E[U(Q ; b) \mid \mathcal{I}]$ whenever $\operatorname{Pr}(\omega=A \mid \mathcal{I})>\tilde{\pi}_{b}\left(\lambda_{i}\right)$ :

$$
\tilde{\pi}_{A}\left(\lambda_{i}\right)=\frac{1-2 \lambda_{i}}{2\left(1-\lambda_{i}\right)}<\frac{1}{2} \quad \text { and } \quad \tilde{\pi}_{Q}\left(\lambda_{i}\right)=\frac{1}{2\left(1-\lambda_{i}\right)}>\frac{1}{2}
$$

${ }^{6}$ While some papers in the literature take this approach, by far the most prevalent is to restrict the analysis to symmetric mixed strategies. To the best of our knowledge, however, there seems to be no clear ranking between these alternative approaches. As it will become clear later, moreover, it is not difficult to see that most (and possibly all) of our results would also continue to hold for symmetric mixed strategy profiles.

${ }^{7}$ Let $h^{j}$ be the history prior to the vote in committee $j$. Since $C_{0}$ is the first to vote, $h^{0}=\emptyset$. Since $C_{1}$ only votes if $A$ defeats $Q$ in the lower committee, feasible histories for individuals in $C_{1}, h^{1}$, are
} 
will sometimes simply write $\sigma_{i}\left(s_{i}, \emptyset\right)$ for $i \in C_{0}$ as $\sigma_{i}\left(s_{i}\right)$. Let $\sigma_{j}\left(s_{j}, h_{j}\right) \equiv\left\{\sigma_{i}\left(s_{i}, h_{j}\right)\right\}_{i \in C_{j}}$, and $\sigma \equiv\left(\sigma_{0}\left(s_{0}\right), \sigma_{1}\left(s_{1}, h_{1}\right)\right)$. We say that a strategy profile $\sigma(\cdot)$ is a voting equilibrium if there exists an $\bar{\alpha}>0$ such that for all $\alpha<\bar{\alpha}$ there exist beliefs $\left\{\mu_{i}^{\alpha}\left(s_{-i} \mid s_{i}, h^{j}\right)\right\}$ such that $\left(\sigma, \mu^{\alpha}\right)$ are a PBE of the game $\Gamma_{\alpha}$ in pure anonymous strategies.

\section{Strategic Voting in Sequential Committees}

We start by formalizing the notions of standard-pivotal and signal-pivotal voting motivations. Consider first a committee $C$ which after history $h$ has the sole authority over whether to approve or reject the proposal (this might be a single committee, in which case $h=\emptyset$, or the receiving committee in a pair of committees moving sequentially, in which case $h=v_{0}$ ). The vote of any member $i \in C$ influences the outcome if and only if $i$ is standard-pivotal in $C$ after $h$; i.e., if and only if $v_{-i}^{h} \in \mathcal{P}_{i}(C, r) \equiv\left\{v_{-i}: t_{-i}\left(v_{-i}\right)=r-1\right\}$. As a result, $i$ 's voting decision is determined by her preference among alternatives as evaluated at the event $\mathcal{P}_{i}(C, r)$ in equilibrium. Then letting

$$
\lambda_{i}^{\mathcal{P}_{i}(C, r)}\left(s_{i}, h\right) \equiv \lim _{\alpha \rightarrow 0} \operatorname{Pr}^{\left(\sigma_{\mid h}, \alpha\right)}\left(\omega_{A} \mid s_{i}, \mathcal{P}_{i}\left(C_{j}, r_{j}\right), h\right),
$$

we say that a strategy profile $\sigma_{\mid h}$ is a voting equilibrium in $C$ if and only if $\sigma_{i}\left(s_{i}\right)=1 \Leftrightarrow$ $\lambda_{i}^{\mathcal{P}_{i}(C, r)}\left(s_{i}, h\right) \geq \pi_{i}$ whenever $\mathcal{P}_{i}(C, r)$ has positive probability under $\sigma_{\mid h}$.

The fundamental difference with respect to voting in a single committee is entirely in the incentives of members of the originating committee, whose vote is guided by a signal-pivotal motivation. For any vote outcome of members of $C_{0}$ other than $i, v_{0-i}$, let $v_{0-i}^{-} \equiv\left(v_{0-i}, v_{i}=-1\right)$ and $v_{0-i}^{+} \equiv\left(v_{0-i}, v_{i}=+1\right)$. We say that an individual $i \in C_{0}$ is signal-pivotal at $k$ if (i) the tally of votes of members of $C_{0}$ other than $i$ equals $k$, and (ii) the proposal loses in the receiving committee if also $i$ votes against the proposal in $C_{0}$, but wins in the receiving committee if $i$ votes in favor of the proposal in $C_{0}$; i.e., if $\left(v_{0-i}, v_{1}^{v_{0-i}^{-}}, v_{1}^{v_{0-i}^{+}}\right) \in \mathcal{S} \mathcal{P}_{i}\left(r_{1}, k\right)$, where

$$
\mathcal{S P}_{i}\left(r_{1}, k\right) \equiv\left\{\left(v_{0-i}, v_{1}^{v_{0-i}^{-}}, v_{1}^{v_{0-i}^{+}}\right): t_{0-i}\left(v_{0-i}\right)=k, t_{1}\left(v_{1}^{v_{0-i}^{-}}\right) \leq r_{1}-2, t_{1}\left(v_{1}^{v_{0-i}^{+}}\right) \geq r_{1}\right\}
$$

We say that an individual $i \in C_{0}$ is signal-pivotal if $\mathcal{S P}_{i}\left(r_{1}, k\right) \neq \emptyset$ for some $k$. Letting $K(\sigma) \equiv\left\{k: \mathcal{S P}_{i}\left(r_{1}, k\right) \neq \emptyset\right\}, \mathcal{S P}_{i}\left(r_{1}\right) \equiv \cup_{k \in K(\sigma)} \mathcal{S P}_{i}\left(r_{1}, k\right)$, we then define:

$$
\lambda_{i}^{\mathcal{S P}}\left(s_{i} ; r_{1}\right) \equiv \sum_{k \in K(\sigma)} \lim _{\alpha \rightarrow 0} \operatorname{Pr}^{(\sigma, \alpha)}\left(\omega_{A} \mid s_{i}, \mathcal{S} \mathcal{P}_{i}\left(r_{1}, k\right)\right) f\left(s_{i}, k\right)
$$

where $\left.f\left(s_{i}, k\right) \equiv \lim _{\alpha \rightarrow 0} \operatorname{Pr}^{\left(\sigma_{0-i}, \alpha\right)}\left(s_{i}, \mathcal{S P}_{i}\left(r_{1}, k\right)\right) \mid s_{i}, \mathcal{S P}_{i}\left(r_{1}\right)\right)$. Then if $\sigma$ is a voting equilibrium, it must be that for all $i \in C_{0}, \sigma_{i}\left(s_{i}\right)=1 \Leftrightarrow \lambda_{i}^{\mathcal{S P}}\left(s_{i} ; r_{1}\right) \geq \pi_{i}$ whenever $\mathcal{S P}_{i}\left(r_{1}\right)$ has positive probability under $\sigma$.

elements of $H^{1}=\left\{v_{0}: t_{0}\left(v_{0}\right) \geq r_{0}\right\}$. By anonymous we mean that $\sigma_{i}\left(s_{i}, h_{0}^{1}\right)=\sigma_{i}\left(s_{i}, h_{1}^{1}\right)$ whenever $h_{1}^{1}$ can be obtained from $h_{0}^{1}$ by only switching elements among lower committee members that play the same strategy. 
We start our analysis in the next section by briefly establishing the key properties of voting equilibria in single committees. For this and throughout the paper it will be useful to express, when possible, posterior probabilities and preferences in terms of a relevant number of positive and negative signals. To this end, we adopt the following conventions throughout the paper. Let $\mathcal{J} \subset C$ be an arbitrary subset of members of a committee $C$. For a given profile of signals $s_{\mathcal{J}} \equiv\left\{s_{i}\right\}_{i \in \mathcal{J}}$, we write $\beta\left(t_{\mathcal{J}}\left(s_{\mathcal{J}}\right)\right) \equiv \operatorname{Pr}\left(\omega=\omega_{A} \mid s_{\mathcal{J}}\right),{ }^{8}$ and let $\rho_{A}$ and $\rho_{Q}$ be the (unique) integers such that $\beta\left(\rho_{b}-1\right) \leq \pi_{b} \leq \beta\left(\rho_{b}\right)$ for $b=A, Q$. The numbers $\rho_{Q}$ and $\left(1-\rho_{A}\right)$ measure the intensity of the bias of conservative (liberal) committee members in terms of the least number of positive (negative) signals that would reverse their policy preference (from our earlier assumption, $\rho_{Q} \geq 2$ and $1-\rho_{A} \geq 2$ ). We let $S_{\mathcal{J}}\left(t ; \sigma_{\mathcal{J}}\right) \equiv\left\{s_{\mathcal{J}}: t_{\mathcal{J}}\left(\sigma_{\mathcal{J}}\left(s_{\mathcal{J}}\right)\right)=t\right\}$, and assuming then that $S_{\mathcal{J}}\left(t ; \sigma_{\mathcal{J}}\right) \neq \emptyset$, define $\tau_{s_{\mathcal{J}}}\left(t, \sigma_{\mathcal{J}}, h_{\mathcal{J}}\right)$ as the sum of the signals of individuals voting informatively in $\mathcal{J}$ that is consistent with a vote tally $t$ following history $h_{\mathcal{J}}$ and given strategy profile $\sigma_{\mathcal{J}}$ if all members of $\mathcal{J}$ are moderates:

$$
\tau_{s_{\mathcal{J}}}\left(t, \sigma_{\mathcal{J}}, h_{\mathcal{J}}\right) \equiv t-\left|\left\{i \in \mathcal{J}: \sigma_{i}\left(s_{i}, h_{\mathcal{J}}\right)=1\right\}\right|+\left|\left\{i \in \mathcal{J}: \sigma_{i}\left(s_{i}, h_{\mathcal{J}}\right)=-1\right\}\right|
$$

Then letting for any event $E, L(E) \equiv \lim _{\alpha \rightarrow 0} \frac{\operatorname{Pr}^{\sigma, \alpha}\left(E \mid \omega_{Q}\right)}{\operatorname{Pr}^{\sigma, \alpha}\left(E \mid \omega_{A}\right)}$, it follows that ${ }^{9}$

$$
\lambda_{i}^{\mathcal{P}_{i}(C, r)}\left(s_{i}, h\right)=\frac{1}{1+L\left(s_{i}\right) L\left(\mathcal{P}_{i}(C, r)\right) L(h)}=\beta\left(s_{i}+\tau_{s_{-i}}\left(r-1 ; \sigma_{-i}\right)+\tau_{0}(h)\right)
$$

, where $\tau_{0}(h)=0$ for single committees $(h=0)$ and $\tau_{0}(h)=\tau_{s_{0}}\left(t_{0}\left(v_{0}\right), \sigma_{0}\right)$ for the case of sequential committees $\left(h=v_{0}\right)$. Therefore, a conservative member of $C$ has an incentive to vote in favor of the proposal if and only if $\beta\left(s_{i}+\tau_{s_{-i}}\left(r-1 ; \sigma_{-i}\right)+\tau_{0}(h)\right) \geq \pi_{Q}$, or equivalently $\tau_{s_{-i}}\left(r-1 ; \sigma_{-i}+\tau_{0}(h)+s_{i} \geq \rho_{Q}\right.$ (similarly for a liberal member, substitute $\left.\rho_{A}\right)$.

\subsection{Basic Results for Single (and Receiving) Committees}

We begin with the simplest case of a committee with common interests; i.e., $\pi_{i}=\pi$ $\forall i \in C$. Here, and in the remainder of the paper, we will follow convention by saying that $i \in C_{\ell}$ votes informatively if $v_{i}(s, \emptyset)=s \forall s$, and that she votes her bias if $v_{i}(s, \emptyset)=b_{i}$ $\forall s$. We start by pointing out a well known result due to Austen-Smith and Banks (1996)

Proposition 1 Consider a committee composed of $n$ members such that $\pi_{i}=\pi \forall i=$ $1, \ldots, n$, operating under a $\frac{r+n}{2}$ majority rule $(z=A$ if and only if $t(v) \geq r)$. Then informative voting for all $i$ is a voting equilibrium iff $r=\rho .^{10}$

${ }^{8}$ The definition of $t_{\mathcal{J}}\left(s_{\mathcal{J}}\right)$ follows the same convention as with votes; i.e., $t_{\mathcal{J}}\left(s_{\mathcal{J}}\right) \equiv \sum_{i \in \mathcal{J}} s_{i}$. Note that $\operatorname{Pr}\left(\omega=\omega_{A} \mid s_{\mathcal{J}}\right)=\operatorname{Pr}\left(\omega=\omega_{A} \mid s_{\mathcal{J}}^{\prime}\right)$ whenever $t_{\mathcal{J}}\left(s_{\mathcal{J}}\right)=t_{\mathcal{J}}\left(s_{\mathcal{J}}^{\prime}\right)$.

${ }^{9}$ Note that if $E_{1}$ and $E_{2}$ are independent, then $\operatorname{Pr}\left(\omega_{A} \mid E_{1}, E_{2}\right)=\left[1+L\left(E_{1}\right) L\left(E_{2}\right)\right]^{-1}$, and that for any $\mathcal{J} \subseteq C, L\left(s_{\mathcal{J}}: \sum_{i \in \mathcal{J}} s_{i}=t\right)=\left(\frac{1-q}{q}\right)^{t}$.

${ }^{10}$ The result is stated for $\rho$ is odd. If $\rho$ is even the condition is $r=\rho+1$. 
The logic behind this result is straightforward. Since all committee members vote informatively, the net number of signals implied by standard pivotality is $\tau_{s_{-i}}\left(r-1, \sigma_{-i}\right)=$ $r-1$ and therefore $\lambda_{i}^{\mathcal{P}_{i}(C, r)}\left(s_{i}\right)=\beta\left(r-1+s_{i}\right)$. Incentive compatibility of $\sigma$ requires $\lambda_{i}^{\mathcal{P}_{i}(C, r)}(-1) \leq \pi \leq \lambda_{i}^{\mathcal{P}_{i}(C, r)}(1)$, and hence $\beta(r-2) \leq \pi \leq \beta(r)$. Then either $r=\rho+1$ (if $\rho$ is even) or $r=\rho$ (if $\rho$ is odd). On the other hand, suppose $r \geq \rho+2$. Then $\lambda_{i}^{\mathcal{P}_{i}(C, r)}(-1)=\beta(r-2) \geq \beta(\rho)$, and hence $i$ has incentive to deviate and vote in favor of the proposal after a negative signal (a similar argument holds if $r \leq \rho-1$ ).

More generally, in the case of a single committee with common interests, equilibria must fall in one of two outcome-relevant classes. First, there exists a class of noninformative equilibria in which the policy outcome is equal to the committee members' bias independently of the private information held by members of the committee. In these equilibria $\sigma_{i}\left(s_{i}\right)=b_{i} \forall s_{i}$ for some decisive majority in $C$ (for all $i$ in some set $\mathcal{J} \subseteq C$ such that $|\mathcal{J}| \geq \frac{r+n}{2}$ ). That this is in fact an equilibrium follows immediately, since in any such strategy profile no individual is ever pivotal, and therefore there are no profitable deviations. ${ }^{11}$ We can typically also construct an asymmetric voting equilibrium in which some committee members vote informatively. Intuitively, here the number of informative votes $k$ is chosen so that for any voting member, the information provided by the equilibrium strategies conditional on him being pivotal exactly compensates the imbalance between the effective rule and $\rho .^{12}$

To see this, consider the following example. Suppose agents are unbaised, so that $\pi=1 / 2$, and that $n=11$ and $r=3$ (the proposal wins if and only if it receives 7 out of 11 votes). Then the strategy profile in which agents 1 to 9 vote informatively and the remaining vote unconditionally in favor of the proposal is a voting equilibrium. Note that according to $\sigma^{*}$, if $i \leq 9$ is pivotal then $t_{-i}\left(\sigma_{-i}\left(s_{-i}\right)\right)=2+\sum_{j \leq 9}^{j \neq i} s_{i}=2$, and as a result $\tau_{-i}\left(2, \sigma_{-i}\right)=0$; i.e., the signals of other players must cancel out. Incentive compatibility is then satisfied since $\lambda_{i}^{\mathcal{P}_{i}(C, r)}\left(s_{i}=-1\right)=\beta(-1)=1-q \leq 1 / 2 \leq q=\beta(1)=\lambda_{i}^{\mathcal{P}_{i}(C, r)}\left(s_{i}=1\right)$ (incentive compatibility for $i \geq 10$ follows from $\tau_{-i}\left(2, \sigma_{-i}\right)=1$ ). This example suggests that different differences between $r$ and $\rho$ correspond to different bounds on the amount of information that can be used in equilibrium. This is in fact generally the case, as Proposition 2 shows:

Proposition 2 Consider a committee composed of $n$ members $i$ such that $\pi_{i}=\pi \forall i$, $\rho(\pi) \leq n$, operating under a $\frac{r+n}{2}$ majority rule. Then (i) there exists a unique voting equilibrium with relevant informative voting if and only if $-(n-r) \leq \rho \leq R$, and (ii) the number of informative votes in this voting equilibrium is decreasing in the difference $|r-\rho|$.

Proof. The proof is in the appendix.

\footnotetext{
${ }^{11}$ Equilibria in which $\sigma_{i}\left(s_{i}\right)=-b_{i} \forall s_{i}$ for all $i$ in some decisive set $\mathcal{J} \subseteq C$ are ruled out due to the existence of partisans.

${ }^{12}$ This is well known. See for example Dekel and Piccione (2000) or Persico (2004). The logic is essentially the same as that in symmetric equilibria in mixed strategies which are often considered in the literature (see for example Feddersen and Pesendorfer (1997)).
} 
The previous results are essentially unchanged - for the relevant population - if, as it is the case in our main model, we introduce two groups with different biases; i.e., there are $n_{Q}$ members with bias $\pi_{i}=\pi_{Q}>q$ and $n-n_{Q}$ members with bias $\pi_{i}=\pi_{A}<1-q$. First, it is immediate to verify that given that $\pi_{Q}>q>1-q>\pi_{A}$, (i) there is no equilibrium in which both conservatives and liberals vote informatively, and that (ii) if in a voting equilibrium $i$ votes informatively and $b_{i} \neq b_{i^{\prime}}$, then $i^{\prime}$ must vote her bias. Note then that informative voting by liberals can only be outcome relevant if liberals are a winning coalition in $C$. Similarly, informative voting by conservatives can only be outcome relevant if conservatives are a blocking coalition in $C$ (we say that individuals in a a subset $\mathcal{J}$ of committee $C$ constitute a winning coalition in $C$, and denote this by $\mathcal{J} \in W(C)$, if $|\mathcal{J}| \geq R$. Alternatively, $\mathcal{J} \subset C$ is a blocking coalition in $C$, or $\mathcal{J} \in B(C)$, if $|\mathcal{J}| \geq n-R+1)$.

Since in any equilibrium with relevant informative voting individuals voting informatively must be conservatives when conservatives are a blocking coalition in $C$ (liberals when instead liberals are a winning coalition in $C$ ), then liberals (conservatives) vote their bias in equilibrium and therefore just act so as to change the number of votes required to pass the proposal. What rests to be determined is then formally equivalent in the case of a single committee - to the analysis in Proposition 2 with majority rule $R_{\mathcal{D}} \equiv \frac{r_{\mathcal{D}}+n_{\mathcal{D}}}{2}$ and population $n_{\mathcal{D}}$, where $r_{\mathcal{D}}=r-n_{A}$ and $n_{\mathcal{D}}=n_{Q}$ when $\mathcal{Q} \in B(C)$ and $r_{\mathcal{D}}=r+n_{Q}$ and $n_{\mathcal{D}}=n_{A}$ when $\mathcal{A} \in W(C)$. The same logic holds, moreover, if the committee is the second of two committees moving sequentially, after we incorporate the information contained in the tally in ex post biases $\rho_{Q}^{\prime}\left(v_{0}, \sigma_{0}\right) \equiv \rho_{Q}-\tau_{s_{0}}\left(v_{0}, \sigma_{0}\right)$ and $\rho_{A}^{\prime}\left(t_{0}\left(v_{0}\right), \sigma_{0}\right) \equiv \rho_{A}-\tau_{s_{0}}\left(t_{0}\left(v_{0}\right), \sigma_{0}\right)$. We summarize the result in the next Proposition. For this, and the remainder of the paper, it will be useful to introduce the following definition.

Definition 1 We will say that the strategy profile of a set of individuals $\mathcal{J} \in C_{j}$ is $k$ informative with liberal (conservative) bias if there exist a labeling of individuals in $\mathcal{J}$ such that all $i \in \mathcal{J}^{I}(k) \equiv\{i \in \mathcal{J}: i \leq k\}$ vote informatively and (ii) all $i \in \mathcal{J}^{N}(k) \equiv$ $\{i \in \mathcal{J}: i>k\}$ vote in favor (against) the proposal.

Then we have shown that, ${ }^{13}$

Proposition 3 (i) Assume $\mathcal{Q}_{1} \in B\left(C_{1}\right)$. Then there exists a unique voting equilibrium with relevant informative voting in $\Gamma\left(v_{0}\right)$ if and only if $-\left(R_{1}^{Q}-r_{1}^{Q}\right) \leq \rho_{Q}^{\prime}\left(v_{0}, \sigma_{0}\right) \leq R_{1}^{Q}$. In this equilibrium, liberals vote their bias and conservatives play a $(i)$ a $\tilde{k}^{0}\left(t_{0}\left(v_{0}\right)\right)$ informative strategy profile with conservative bias if $r_{1}^{Q} \leq \rho_{Q}^{\prime}\left(v_{0}, \sigma_{0}\right)$ and (ii) a $\tilde{k}^{1}\left(t_{0}\left(v_{0}\right)\right)$ informative strategy profile with liberal bias if $r_{1}^{Q}>\rho_{Q}^{\prime}\left(v_{0}, \sigma_{0}\right)$, where $\tilde{k}^{0}\left(t_{0}\left(v_{0}\right)\right) \equiv n_{1}^{Q}-$ $\left[\rho_{Q}^{\prime}\left(v_{0}, \sigma_{0}\right)-r_{1}^{Q}\right]$ and $\tilde{k}^{1}\left(t_{0}\left(v_{0}\right)\right)=n_{1}^{Q}-\left[r_{1}^{Q}-\rho_{Q}^{\prime}\left(v_{0}, \sigma_{0}\right)\right]+1$.

(ii) When instead $\mathcal{A}_{1} \in W\left(C_{1}\right)$, there exists a unique voting equilibrium with relevant informative voting in $\Gamma\left(v_{0}\right)$ if and only if $-\left[\left(R_{1}-1\right)-r_{1}\right] \leq 1-\rho_{A}^{\prime}\left(v_{0}, \sigma_{0}\right) \leq\left[R_{1}^{A}-\left(r_{1}^{A}-1\right)\right]$.

\footnotetext{
${ }^{13}$ The result is stated for the second of two committees moving sequentially. For a single committee, simply write $\rho_{\mathcal{D}}^{\prime}\left(v_{0}, \sigma_{0}\right)=\rho_{\mathcal{D}}$ and ignore all references to the history.
} 
In this equilibrium, conservatives vote their bias and liberals play a (i) a $\tilde{k}^{0}\left(t_{0}\left(v_{0}\right)\right)$ informative strategy profile with conservative bias if $-\left[1-\rho_{A}^{\prime}\left(v_{0}, \sigma_{0}\right)\right] \geq r_{1}^{A}$ and (ii) a $\tilde{k}^{1}\left(t_{0}\left(v_{0}\right)\right)$-informative strategy profile with liberal bias if $-\left[1-\rho_{A}^{\prime}\left(v_{0}, \sigma_{0}\right)\right]<r_{1}^{A}$, where $\tilde{k}^{0}\left(t_{0}\left(v_{0}\right)\right) \equiv n_{1}^{A}-\left[\left(1-\rho_{A}^{\prime}\left(v_{0}, \sigma_{0}\right)\right)-r_{1}^{A}\right]$, and $\tilde{k}^{1}\left(t_{0}\left(v_{0}\right)\right)=n_{1}^{A}-\left[r_{1}^{A}-\left(1-\rho_{A}^{\prime}\left(v_{0}, \sigma_{0}\right)\right)\right]+1$.

Note, in particular, that the number of informative votes in the receiving committee is decreasing in the difference between the effective hurdle $r_{\mathcal{D}}^{\prime}$ that individuals voting informatively in the receiving committee must surpass, and their ex post bias $\rho_{\mathcal{D}}^{\prime}\left(v_{0}, \sigma_{0}\right)$ after observing the public signal $\tau_{s_{0}}\left(t_{0}, \sigma_{0}\right)$.

Proposition 3 completely characterizes informative strategic voting in a single committee. We turn next to the analysis of voting in sequential committees. Note that as for single committees, here the uninformative strategy profile in which individuals in each committee vote unconditionally for the alternative when liberals are a winning coalition in their committee $\left(\mathcal{A}_{j} \in W\left(C_{j}\right)\right)$ and for the status quo when conservatives are a blocking coalition in their committee $\left(\mathcal{Q}_{j} \in B\left(C_{j}\right)\right)$ is a voting equilibrium. In this voting equilibrium, however, there is no transmission of information (or even more, no use of information of any sort). We focus from here on on voting equilibria in which the equilibrium outcome is responsive to private information; i.e., given $\sigma$, there exist two realizations of private signals $s, s^{\prime} \in S$ such that $z=A$ under $s$ but $z=Q$ under $s^{\prime}$.

The equilibrium outcome can be responsive to private information in (broadly) three different ways, and we categorize classes of equilibria accordingly. In a voting equilibrium with relevant one sided informative voting, all informative voting occurs in one committee (either in the originating or the receiving committee). When this happens in the receiving committee, members of the originating committee must (collectively) approve the proposal unconditionally (as otherwise the proposal would never reach the receiving committee). When instead informative voting occurs only in the originating committee, members of the receiving committee can act conditional on the outcome of this vote, but only bluntly, approving the proposal unconditionally for some outcomes and "killing" it unconditionally for others. Finally, the equilibrium outcome can be responsive to private information in both committees. Here not only does the second committee acts conditional on the outcome of the vote in the originating committee, but more specifically the number of individuals voting informatively will be a function of the voting outcome in $C_{0}$. We begin in the next section by tackling voting equilibria in which all informative voting occurs in the originating committee.

\subsection{An Endogenous Majority Rule}

We consider here voting equilibria in which all informative voting occurs in the originating committee, which for reasons that will be apparent shortly we label endogenous majority rule (EMR) voting equilibria. In equilibria of this class, the second committee acts only to modify the hurdle that the alternative has to surpass in the first committee to defeat the status quo in equilibrium (from, say, a simple majority to a two thirds majority), 
in such a way as to induce informative voting by some of its members in equilibrium: the endogenous threshold introduced by the receiving committee "replicates" the effect of the optimal fixed rule in Austen-Smith and Banks (1996). ${ }^{14}$

The main idea is the following: Suppose that the receiving committee kills the proposal - independently of the realization of private information - for all voting outcomes in the lower committee $v_{0}$ with tally $t_{0}\left(v_{0}\right)$ below some critical number $\theta_{0}$, and unconditionally approves the proposal otherwise. For members of the initiating committee, this situation is equivalent to a unicameral system with a modified majority rule $\theta_{0}$. In particular, $\lambda_{i}^{\mathcal{S P}}\left(s_{i} ; r_{1}\right)$ boils down to

$$
\lambda_{i}^{\mathcal{S P}}\left(s_{i} ; r_{1}\right)=\beta\left(\tau_{s_{0-i}}\left(\theta_{0}, \sigma_{0-i}^{*}\right)+s_{i}\right)
$$

It follows from this and the results in section 4.1 that if, given the information provided by the outcome of the vote and the voting strategy profile in the originating committee, we can induce members of the relevant decisive coalition in $C_{1}$ to choose the cutoff $\theta_{0}$ in such a way that the ensuing endogenous majority rule for individuals voting informatively in the originating committee is equal to $\rho_{Q}$ (if they are conservatives) or $\left(1-\rho_{A}\right)$ (for liberals), then these individuals would have an incentive to vote informatively in the first place. Theorem 1 shows that this can always be done when conservatives are a blocking coalition in the receiving committee provided that the number of conservatives and liberals in $C_{0}$ is large enough. In contrast to this positive result, the second part of Theorem 1 shows that this is not possible when liberals control the second committee.

Theorem 1 There does not exist an EMR voting equilibrium in which all liberals in the originating committee vote informatively. There exists an EMR voting equilibrium in which all conservatives in the originating committee vote informatively if and only if $\mathcal{Q}_{1} \in B\left(C_{1}\right)$ and the number of liberals and conservatives in the originating committee is sufficiently large (i.e., if and only if $n_{0}^{Q}>\rho_{Q}$ and $\left.n_{0}^{A} \geq\left(r_{0}-1\right)-\rho_{Q}\right)^{15}$

Proof of Theorem 1. Follows from Lemmas 1 and 2 in the appendix.

The intuition for this contrast is as follows. Suppose first that liberals control the receiving committee, and assume that in equilibrium all liberals in $C_{0}$ vote informatively.

\footnotetext{
${ }^{14}$ Lemma 3 in the appendix shows that all voting equilibria in which all informative voting occurs in the originating committee must be EMR voting equilibria.

${ }^{15}$ To see the role of these conditions, note that when conservatives vote informatively and liberals vote their bias in the originating committee, these are equivalent to requiring that there exist tallies $t_{0} \geq r_{0}$ and $t_{0}^{\prime} \leq n_{0}$ such that $t_{0}=t_{0}\left(\sigma_{0}\left(s_{0}\right)\right)$ and $t_{0}^{\prime}=t_{0}\left(\sigma_{0}\left(s_{0}^{\prime}\right)\right)$ for some $s_{0}$ and $s_{0}^{\prime}$ and $\tau_{s_{0}}\left(t_{0}, \sigma_{0}\right)<$ $\rho_{Q}<\tau_{s_{0}}\left(t_{0}^{\prime}, \sigma_{0}\right)$. First, since only conservatives in the originating committee are voting informatively, $n_{0}^{Q}>\rho_{Q}$ assures that the maximum informativeness of the aggregate public signal for individuals in the receiving committee is larger than the bias of conservatives. Second, $n_{0}^{A} \geq\left(r_{0}-1\right)-\rho_{Q}$ assures that the $R_{0}$-majority rule is not excessively demanding relative to the size of liberals in the originating committee so as to make any tally that passes this threshold an overwhelming positive signal for conservatives in the receiving committee. Note in particular that this condition is never binding for simple majority rule, in which case it is enough that $n_{0}^{Q}>\rho_{Q}$.
} 
The relevant incentive compatibility constraint is that of liberals voting against their bias for "low tallies" (i.e., for $v_{0}: t_{0}\left(v_{0}\right) \leq \theta_{0}-1$ ). Since the inference of an individual $i$ in the originating committee cannot be too different than that of a member of the receiving committee in equilibrium (see the proof for details), members of the originating committee voting informatively must be liberals too. Now since in equilibrium conservatives can't vote against their bias, this means that they must be voting their bias. But this is not possible either, for in this case every positive net tally would carry favorable information for the proposal, and the liberal winning coalition (which is already biased in favor of the proposal), would never have an incentive to vote against it.

It is now apparent why it is not a problem to construct a EMR voting equilibrium when conservatives are a blocking coalition in the receiving committee. The same logic suggests, moreover, that when liberals are a winning coalition in the receiving committee, it might be possible to have an EMR voting equilibrium in which some liberals in the originating committee vote informatively: the asymmetry in the strategy profile of liberals in the originating committee solves the previous problem by making unnecessary that conservatives in $C_{0}$ vote against their bias in order for some positive tally to transmit unfavorable information for the proposal. A generalized version of Theorem 1 shows that under some conditions this is in fact possible. This requires, however, liberals to be a winning coalition not only in the receiving but also in the originating committee as well. Moreover, as Theorem 2 shows, the number of informative votes can never be larger than the majority premium of liberals in $C_{0}$. The theorem also shows that for an EMR voting equilibrium where some conservatives vote informatively we need only assure that conservatives are a blocking coalition in the receiving committee and that the number of conservatives in $C_{0}$ is larger than $\rho_{Q}$.

Theorem 2 (i) Assume $\mathcal{Q}_{1} \in B\left(C_{1}\right)$ and let $k>\rho_{Q}$ be given. If the number of conservatives in the originating committee is sufficiently large, there exists an EMR voting equilibrium with $k$ informative votes; (ii) if $\mathcal{A}_{1} \in W\left(C_{1}\right)$ an EMR voting equilibrium exists only if liberals are also a winning coalition in the originating committee, and the number of informative votes in any EMR voting equilibrium is bounded above by the majority premium of liberals in the originating committee.

Proof of Theorem 2. Follows from Lemmas 1 and 2 in the appendix.

It follows from Theorems 1 and 2 that there are significant behavioral differences in EMR voting equilibria when liberals are or are not a winning coalition in the receiving committee. But there is a also a difference in terms of efficiency of equilibria in the two cases. While EMR voting equilibria are inherently inefficient - since no information from members of the receiving committee influences the choice of policy - the most informative EMR voting equilibria when conservatives are a blocking coalition in the receiving committee selects the "right" alternative (for conservatives) almost surely as the number of conservatives and liberals in the originating committee is sufficiently high ("right" here means the alternative that conservatives would prefer if all the private 
information were made public). This is not the case, however, when liberals are a winning coalition in the receiving committee, as the number of informative votes in the most informative EMR voting equilibria is bounded above by the majority premium of liberals in the originating committee (Proposition 4 in the appendix makes this point formally).

\subsection{Delegation to the Receiving Committee}

In EMR voting equilibria, the role of members of the receiving committee is limited to modifying the effective majority rule faced by members of the initiating committee. The receiving committee approves the proposal when the tally of votes in the originating committee carries sufficient favorable information for the proposal, and rejects it otherwise, but does not use the private information of its members. While under some conditions this will lead the relevant decisive majority to achieve payoffs close to the maximum possible attainable payoffs under perfect information, in other cases it will lead to mistakes occurring with high probability. We show in this section that under some conditions the relevant majority can do better than in the most informative EMR voting equilibrium by simply delegating all relevant decision making to the receiving committee.

The main intuition is straightforward. Suppose for concreteness that both committees are entirely composed by liberals, and that the first committee is small in size (say it has three members) and the second committee is large. Then a EMR voting equilibrium wastes a large amount of information, and incurs in mistakes with very high probability. All committee members would do better in this case if members of the first committee delegated the decision to members of the second committee by voting uninformatively in favor of the proposal. Facing an uninformative history, members of the receiving committee could play a strategy profile with relevant informative voting that allows much more information to be of use (all but $\left|\left(1-\rho_{A}\right)-r_{1}\right|$ members could vote informatively). In general, the ranking between equilibria with one-sided relevant informative voting will depend on the composition of committees. For simplicity, we focus here on the case of simple majority rule. We show that for simple majority rule, the relevant comparison is between the majority premium of liberals in each committee when liberals are a winning coalition in the receiving committee, but between the majority premium of conservatives in the receiving committee and the total number of conservatives in the originating committee otherwise.

Consider first the case in which liberals don't have a majority in the receiving committee. Let $\sigma_{0}$ be an uninformative strategy profile in the originating committee with associated vote outcome $v_{0}$ and tally $t_{0}\left(v_{0}\right) \geq 1$. Then Proposition 3 implies that if (and only if) the bias of conservatives $\rho_{Q}$ is lower than the effective majority rule for conserva-

tives when liberals vote in favor of the proposal $\left(R_{1}^{Q}\right)$, then there exists a unique voting equilibrium with relevant informative voting in the continuation $\Gamma\left(v_{0}\right)$, in which liberals vote their bias and conservatives play a $k$-informative strategy profile with conservative bias, with $k=n_{1}^{Q}-\left[\rho_{Q}^{\prime}\left(v_{0}, \sigma_{0}\right)-r_{1}^{Q}\right]=n_{1}^{Q}-\left[\rho_{Q}-\left(1-n_{1}^{A}\right)\right]$ informative votes. But then note that since passage of the proposal implies that $\sum_{i \in \mathcal{Q}_{1}^{I}} s_{i} \geq \rho_{Q}$, there exists a voting 
equilibrium in which members of the originating committee vote in favor of the proposal uninformatively, and on the equilibrium path members of the receiving committee play the voting equilibrium with relevant informative voting. ${ }^{16}$ The comparison between this and the most informative EMR voting equilibrium is now immediate. In essence, the comparison hinges between the size of the population possibly voting informatively in a EMR voting equilibria $\left(n_{0}^{Q}\right)$ and the majority premium of conservatives in $C_{1}, n_{1}^{Q}-n_{1}^{A}$.

Remark 1 Suppose that both committees operate under simple majority rule, and that $\mathcal{Q}_{1} \in B\left(C_{1}\right)$. (i) If the number of conservatives in $C_{0}$ is larger than the majority premium of conservatives in $C_{1}$ (i.e., $n_{0}^{Q}>n_{1}^{Q}-n_{1}^{A}$ ), then whenever there exists a voting equilibrium with informative voting in $C_{1}$ only, $\sigma^{*}$, there also exists a EMR voting equilibrium $\sigma^{* *}$ that improves the welfare of conservatives vis a vis $\sigma^{*}$; (ii) for any majority premium of conservatives in $C_{1}$ for which there exists a voting equilibrium with informative voting in $C_{1}$ only, there is a low enough $n_{0}^{Q}\left(n_{0}^{Q}<n_{1}^{Q}-n_{1}^{A}+1-\rho_{Q}\right)$ such that if a EMR voting equilibrium $\sigma^{* *}$ exists, it is dominated by $\sigma^{*}$ in terms of conservatives' welfare.

Suppose on the other hand that liberals are a winning coalition in the receiving committee. Then again assuming that $\sigma_{0}$ is uninformative (and letting $v_{0}=\sigma_{0}\left(s_{0}\right)$ be the associated voting outcome), Proposition 3 implies that there exists a unique voting equilibrium with relevant informative voting in the continuation $\Gamma\left(v_{0}\right)$ if and only if $\left(1-\rho_{A}\right) \leq R_{1}^{A}-r_{1}^{A}+1$, and in this equilibrium conservatives vote their bias and liberals play a $k$-informative strategy profile with liberal bias, $k=n_{1}^{A}-\left[r_{1}^{A}-\left(1-\rho_{A}^{\prime}\left(v_{0}, \sigma_{0}\right)\right)\right]+1=$ $n_{1}^{A}-\left[\left(1+n_{1}^{Q}\right)-\left(1-\rho_{A}\right)\right]+1$. Here, however, inducing conservatives in the originating committee to unconditionally "defer" the decision to the receiving committee is not always possible (at least not when they can block the passage of the proposal in $C_{0}$ ). A necessary and sufficient condition for this is that the bias of both conservatives and liberals are small enough relative to the size of the receiving committee. However, when liberals are a winning coalition in the receiving committee, whenever there exists an EMR voting equilibrium, liberals must control the originating committee as well (and the number of informative votes in EMR voting equilibria is bounded above by the majority premium in the originating committee). As a result, the relevant comparison now is entirely between majority premiums in each committee:

Remark 2 Suppose that both committees operate under simple majority rule, and that $\mathcal{A}_{1} \in W\left(C_{1}\right)$. (i) If the majority premium of liberals in $C_{0}$ is larger than in $C_{1}$ (i.e., $\left.n_{0}^{A}-n_{0}^{Q}>n_{1}^{A}-n_{1}^{Q}\right)$, then whenever there exists a voting equilibrium with informative voting in $C_{1}$ only, $\sigma^{*}$, there also exists a EMR voting equilibrium $\sigma^{* *}$ that improves the welfare of conservatives vis a vis $\sigma^{*}$. Conversely, if $n_{0}^{A}-n_{0}^{Q} \leq n_{1}^{A}-n_{1}^{Q}$, then whenever there exists a EMR voting equilibrium $\sigma^{* *}$, there also exists a voting equilibrium with

\footnotetext{
${ }^{16}$ Suppose that $C_{1}$ members treat any deviation from $\sigma_{0}$ as uninformative (note that this should always be the case for conservatives), and play the voting equilibrium with relevant informative voting following any $v_{0}$ such that $t_{0}\left(v_{0}\right) \geq r_{0}=1$. Note that $i \in C_{0}$ 's vote can only be outcome relevant if there are $\frac{n_{0}-1}{2}$ conservative partisans - which in particular is not possible if $\mathcal{A}_{0} \in W\left(C_{0}\right)$ - so that $t_{0-i}=0$. But then $i$ 's vote changes the outcome if and only if almost surely $\sum_{i=1}^{k} s_{i} \geq \rho_{Q}$, and hence no individual in $C_{0}$ prefers to deviate and vote against the proposal.
} 
informative voting in $C_{1}$ only, $\sigma^{*}$, which improves the welfare of conservatives vis a vis $\sigma^{* *}$.

\subsection{Relevant Informative Voting in Both Committees}

We consider next candidate equilibria in which some members of both committees vote informatively, restricting ourselves to profiles that are monotonically responsive. Say that the tally $t_{0}\left(\sigma_{0}\left(s_{0}\right)\right)$ is informative if $(i) \sigma_{i}(s, \emptyset)=s$ for all $s$ for some $i \in C_{0}$, and (ii) $\exists$ $s_{0}$ such that $t_{0}\left(\sigma_{0}\left(s_{0}\right)\right) \geq r_{0}$.

Definition 2 We say that a strategy profile $\sigma^{*}$ is a monotonically responsive voting equilibrium if (i) it is a voting equilibrium, (ii) $t_{0}\left(\sigma_{0}^{*}\left(s_{0}\right)\right)$ is informative, and (iii) $\forall s_{1}$, $t_{0}\left(\sigma_{0}^{*}\left(s_{0}\right)\right) \geq t_{0}\left(\sigma_{0}^{*}\left(s_{0}^{\prime}\right)\right) \Rightarrow t_{1}\left(\sigma^{*}\left(s_{1}, s_{0}\right)\right) \geq t_{1}\left(\sigma^{*}\left(s_{1}, s_{0}^{\prime}\right)\right)$, with strict inequality for some $s_{1}, s_{0}$ and $s_{0}^{\prime}$.

Voting equilibria with relevant two sided informative voting have two properties. First, the probability of passage of the proposal in the second committee is increasing in the tally of votes in favor of the proposal in the first committee. Essentially, the proposal must go through two relevant tests to succeed, and the level of difficulty of the second test decreases the more favorable the result of the initial test. Second, the number of informative votes in the receiving committee is decreasing in the difference between the effective hurdle $r^{\prime}$ that these individuals voting informatively in the receiving committee must surpass and their ex post bias $\rho^{\prime}$, after observing the public signal $\tau_{s_{0}}\left(t_{0}, \sigma_{0}\right)$.

As before, here the problem of members of the receiving committee is equivalent to that of members of a single committee (after observing a public signal $\tau_{s_{0}}\left(t_{0}, \sigma_{0}\right)$ ): $i \in C_{1}$ 's vote is influential to the outcome if and only if it is pivotal in the traditional sense of being the decisive vote in a divided committee (if it is standard-pivotal). The problem of members of the originating committee, however, changes significantly: $i \in C_{0}$ 's vote matters not only according to whether it is necessary to pass the proposal or not in the initiating committee, but also as a way to transmit information to members of the receiving committee (the signal-pivotal motive). As a result, while in a voting equilibrium with one sided informative voting in the originating committee there is only one way of being pivotal (absent name-flipping), when individuals in the receiving committee also vote informatively this is generically no longer the case; i.e., the set $K(\sigma) \equiv\{k$ : $\left.\mathcal{S P}_{i}\left(r_{1}, k\right) \neq \emptyset\right\}$ has typically more than one element.

Our first goal is to exploit the implications of the incentive compatibiliy constraints of individuals in both committees to restrict the set of strategy profiles that can possibly be voting equilibria. Recall first (see Proposition 3 ) that for any voting outcome $v_{0}$ in the originating committee, the number of informative votes in the receiving committee in the unique equilibrium with relevant informative voting in the continuation game $\Gamma\left(v_{0}\right)$ is decreasing in the difference between the effective hurdle $r^{\prime}$ that individuals voting 
informatively must surpass, and their ex post bias $\rho^{\prime}$ after observing the public signal $\tau_{s_{0}}\left(t_{0}, \sigma_{0}\right)$.

Now, by monotonicity, if there is relevant informative voting in the receiving committee following voting outcomes $v_{0}$ and $v_{0}^{\prime}$ in the originating committee, then there must also be relevant informative voting in $C_{1}$ following $v_{0}^{\prime \prime}$ whenever $t_{0}\left(v_{0}\right) \leq t_{0}\left(v_{0}^{\prime \prime}\right) \leq t_{0}\left(v_{0}^{\prime}\right)$. Furthermore, since the only relevant difference for individuals in the receiving committee between voting outcomes $v_{0}^{\prime}$ and $v_{0}^{\prime \prime}$ with adjacent tallies $\left(t_{0}\left(v_{0}^{\prime \prime}\right)=t_{0}\left(v_{0}^{\prime}\right)+2\right)$ lies almost surely in the (different) realization of the signal of a member of $C_{0}$ voting informatively, then the number of individuals voting informatively in the respective continuation games in the receiving commitee, $\tilde{k}\left(t_{0}\left(v_{0}\right)\right)$, must increase or decrease linearly with $t_{0}$ and in particular must satisfy $\left|\tilde{k}\left(t_{0}\left(v_{0}^{\prime \prime}\right)\right)-\tilde{k}\left(t_{0}\left(v_{0}^{\prime}\right)\right)\right|=2$. In fact, as Proposition 3 also shows, if $\tilde{k}\left(t_{0}\left(v_{0}\right)\right)$ is increasing at some $t$ and decreasing at $t^{\prime}$, then $t<t^{\prime}$, with individuals initially switching from voting against the proposal unconditionally to voting informatively - for tallies in some range $\underline{\theta}_{0}<t_{0}<\theta_{0}$ - and then from voting informatively to voting in favor of the proposal in some range $\theta_{0}<t_{0}<\bar{\theta}_{0}$.

As a result, in any voting equilibrium with relevant two-sided informative voting, the likelihood of the proposal defeating the status quo is not a step function as under an EMR voting equilibrium, but is instead strictly increasing with the tally in the originating committee (for voting outcomes $v_{0}$ in $C_{0}$ such that $\underline{\theta}_{0}<t_{0}\left(v_{0}\right)<\bar{\theta}_{0}$ ). We then have,

Proposition 4 Suppose $\sigma$ is a MR voting equilibrium with relevant two-sided informative voting. Then there exist $\underline{\theta}_{0}, \theta_{0}$ and $\bar{\theta}_{0}\left(r_{0}-1 \leq \underline{\theta}_{0} \leq \theta_{0} \leq \bar{\theta}_{0} \leq n_{0}+1\right)$, and $\tilde{k}^{0}\left(t_{0}\right)$, $\tilde{k}^{1}\left(t_{0}\right)$ such that: (i) individuals in a winning or blocking coalition in $C_{1}$ play a $\tilde{k}^{0}\left(t_{0}\left(v_{0}\right)\right)$ informative strategy profile with conservative bias for all $v_{0}: \underline{\theta}_{0}+1 \leq t_{0}\left(v_{0}\right) \leq \theta_{0}-1$ and a $\tilde{k}^{1}\left(t_{0}\left(v_{0}\right)\right)$-informative strategy profile with liberal bias for $v_{0}: \theta_{0}+1 \leq t_{0}\left(v_{0}\right) \leq \bar{\theta}_{0}-1$. Moreover, $\tilde{k}^{0}\left(t_{0}+2\right)=\tilde{k}^{0}\left(t_{0}\right)+2$, and $\tilde{k}^{1}\left(t_{0}+2\right)=\tilde{k}^{1}\left(t_{0}\right)-2$; (ii) a decisive majority of individuals in $C_{1}$ votes uninformatively for $Q$ if $t_{0}\left(v_{0}\right)<\underline{\theta}_{0}$ or $A$ if $t_{0}\left(v_{0}\right)>\bar{\theta}_{0}$.

Proposition 4 offers a partial characterization of a voting equilibrium with relevant two-sided informative voting, assuming that such a voting equilibrium exists. But is it at all possible to have two-sided relevant informative voting in equilibrium? We show below that this is indeed the case. In particular, we show that for an equilibrium of this class to exist it is sufficient that conservatives form a blocking coalition in the receiving committee and that the number of conservatives in the originating committee is sufficiently large. We also show, however, that the large number of conservatives in the originating committee is only a sufficient condition, and that there also exists a voting equilibrium with relevant two sided informative voting in which each of a small number of conservatives in the originating committee (but at least $\rho_{Q}$ ) votes informatively when liberals are sufficiently numerous (moreover, this strategy profile remains an equilibrium when voting within each committee is allowed to be sequential as well).

To see the intuition for this result, assume then that conservatives are a blocking coalition in the receiving committee, and consider the problem of an individual $i \in C_{0}$ 


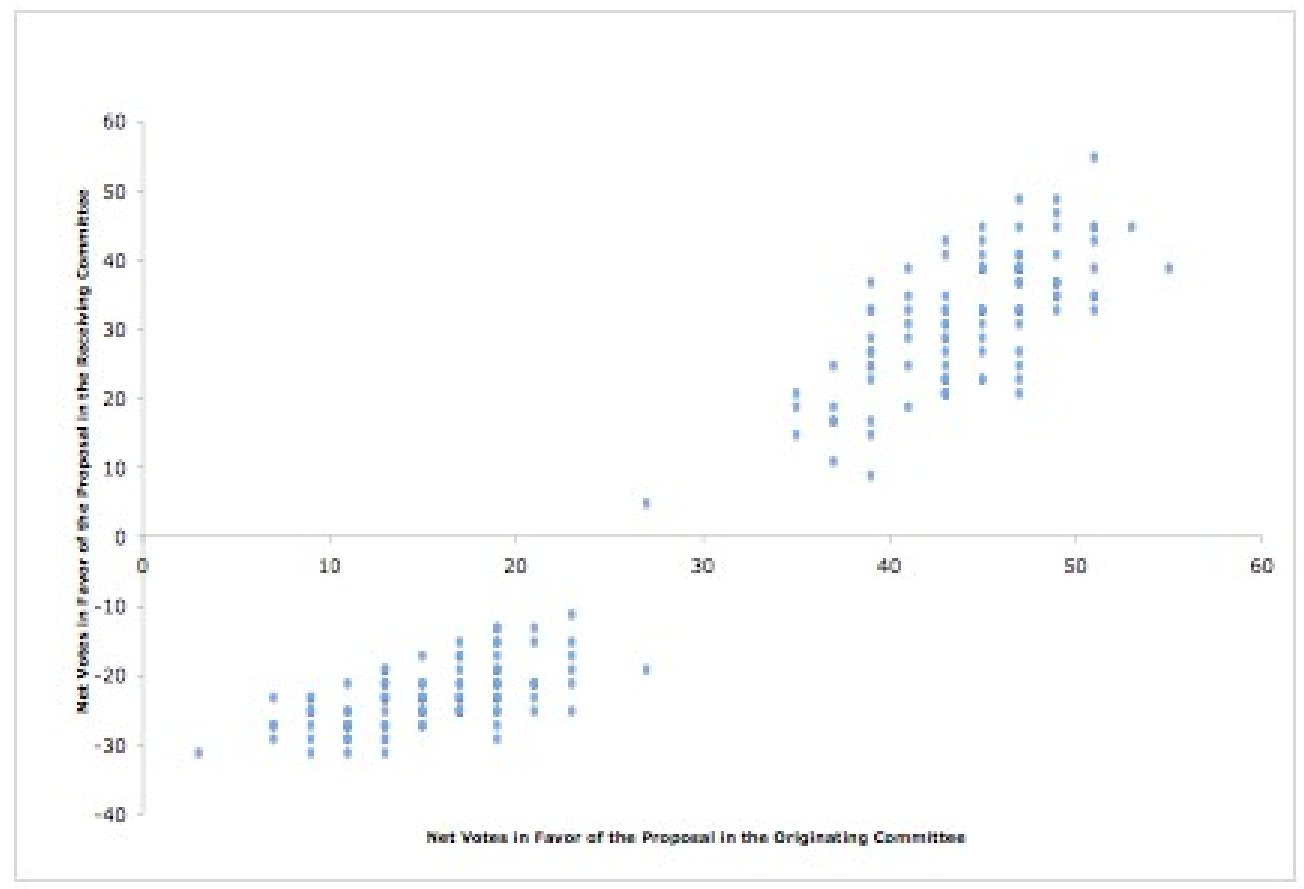

Figure 1: Two-Sided Relevant Informative Voting

voting informatively in a voting equilibrium with relevant two-sided informative voting. Fix any voting outcome of the remaining members of the initiating committee such that $\underline{\theta}_{0}+2 \leq t_{0-i}\left(v_{0-i}\right) \leq \theta_{0}-2$. For $i$ 's vote to be payoff relevant, it must be that the proposal loses against the status quo in $\Gamma\left(v_{0-i}^{-}\right)$but defeats it in $\Gamma\left(v_{0-i}^{+}\right)$. But from Proposition 4 , this must be due to the vote of two members of the receiving committee who vote uninformatively against the proposal in $\Gamma\left(v_{0-i}^{-}\right)$but vote informatively in $\Gamma\left(v_{0-i}^{+}\right)$, in response to the (almost sure) reversal of a negative signal in the originating committee leading from $t_{0-i}\left(v_{0-i}\right)-1$ to $t_{0-i}\left(v_{0-i}\right)+1$. This implies (as we show in Lemma 6 in the appendix) that here $\beta\left(\rho_{Q}-1+s_{i}\right) \leq \lim _{\alpha \rightarrow 0} \operatorname{Pr}^{(\sigma, \alpha)}\left(\omega_{A} \mid s_{i}, \mathcal{S P}_{i}\left(r_{1}, t_{0-i}\left(v_{0-i}\right)\right)\right) \leq \beta\left(\rho_{Q}+s_{i}\right)$, and therefore that, conditional on any $v_{0-i}$ with a tally in $\left(\underline{\theta}_{0}, \theta_{0}\right)$, a conservative in the originating committee has incentives to vote informatively (and therefore also a liberal in the originating committee to vote his bias).

The same is true therefore in expectation if all possible voting outcomes in the originating committee have tallies in $\underline{\theta}_{0}+2 \leq t_{0-i}\left(v_{0-i}\right) \leq \theta_{0}-2$. So suppose that in fact liberals in $C_{0}$ vote their bias, and (all) conservatives in $C_{0}$ vote informatively. Proposition 3 showed that there can only exist a voting equilibrium with informative voting in a continuation $\Gamma\left(v_{0}\right)$ if $\rho_{Q}^{\prime}\left(v_{0}, \sigma_{0}\right) \leq R_{1}^{Q}$, or equivalently $\tau_{s_{0}}\left(t_{0}\left(v_{0}\right), \sigma_{0}\right) \geq \rho_{Q}-\left(\frac{n_{1}^{Q}-n_{1}^{A}+r_{1}}{2}\right)$. Lemma 5 in the appendix shows that if given $\sigma_{0}$, this inequality holds as an equality for a $t_{0}\left(v_{0}\right)$ such that $r_{0}-1 \leq t_{0}\left(v_{0}\right) \leq n_{0}-1$, then we can always find $\underline{\theta}_{0}$ such that no individual in $C_{0}$ would want to deviate from playing according to $\sigma_{0}$ conditional on knowing $t_{0-i}\left(v_{0-i}\right)=\underline{\theta}_{0}$. If in addition $\rho_{Q}^{\prime}\left(v_{0}, \sigma_{0}\right) \geq r_{1}^{Q}$ for all $v_{0}$, or equivalently $\tau_{s_{0}}\left(n_{0}, \sigma_{0}\right)-\rho_{Q} \leq n_{1}^{A}-r_{1}$, then Proposition 3 shows that for all feasible voting out- 
comes in $C_{0}$, if there is a voting equilibrium in $\Gamma\left(v_{0}\right)$ with informative voting, it must be that conservatives in $C_{1}$ play a k-informative strategy profile with conservative bias (i.e., for all feasible $v_{0-i}, t_{0-i}\left(v_{0-i}\right) \leq \theta_{0}-1$ ). In terms of the composition and size of the committees, the previous conditions can be written as:

$$
0 \leq n_{0}^{Q}-\rho_{Q} \leq n_{1}^{A}-r_{1} \text { and } n_{0}^{A} \geq \frac{n_{1}^{Q}-n_{1}^{A}+r_{1}}{2}+\left(r_{0}-\rho_{Q}\right)
$$

Our previous argument then suggests that if conditions (5) hold, there will exist a voting equilibrium with relevant two-sided informative voting in which all conservatives in the originating committee vote informatively. Note moreover, that we have argued above that conservatives in the originating committee have incentives to vote informatively not only in expectation but also conditional on any $v_{0-i}$ with a tally in $\left(\underline{\theta}_{0}, \theta_{0}\right)$. But together with the results of Dekel and Piccione (2000) this directly implies that the previous strategy profile is also a voting equilibrium for sequential voting within each committee (as are voting equilibria with one sided informative voting). To be more precise, say that a voting equilibrium is robust to sequential voting within each committee if every $T$-period voting game has a Nash Equilibrium in which for any history of votes observed prior to the play of any individual $i$, the vote choice conditional on $s_{i}$ is the same as that prescribed by $\sigma$. Then we have

Theorem 3 Suppose that $\mathcal{Q}_{1} \in B\left(C_{1}\right)$ and that conditions (5) hold. Then

(i) there exists a voting equilibrium with relevant two-sided informative voting that is robust to sequential voting within each committee. Moreover,

(ii) there exists an equilibrium of this class in which all conservatives in the originating committee vote informatively. In any such equilibrium, conservatives in the receiving committee vote against the proposal unconditionally if $t_{0}\left(v_{0}\right)<\underline{\theta}_{0}$ and otherwise play a $\left[\bar{k}+t_{0}\left(v_{0}\right)\right]$-informative strategy profile with conservative bias. ${ }^{17}$

Proof. The proof is in the appendix.

Theorem 3 has the following immediate implication:

Corollary 1 Let $\sigma^{*}$ be the strategy profile of Theorem 3, and $\mu\left(t_{0} ; \sigma\right)=\operatorname{Pr}\left(z_{1}=A \mid t_{0} ; \sigma\right)$ denote the conditional probability of the proposal being accepted conditional on the tally $t_{0} \geq r_{0}$ in $C_{0}$ (given $\sigma$ ). Then (i) $\mu\left(t_{0} ; \sigma^{*}\right)<1$ for all $t_{0} \geq r_{0}$, and (ii) $\mu\left(t_{0} ; \sigma^{*}\right)$ is strictly increasing in $t_{0}$ for all $t_{0}>\underline{\theta}_{0}$.

\footnotetext{
${ }^{17} \mathrm{~A}$ similar strategy profile in which some conservatives vote uninformatively in favor of the proposal - reducing therefore the probability of incorrectly killing the proposal in the originating committee - can shown to be a voting equilibrium in environments (composition of committees) in which the profile in Theorem 3 is not; i.e., even when conditions (5) do not hold. This follows immediately from Theorem 3 after noting that uninformative conservatives would not have profitable deviations, since then our previous analysis applies with the relabeling $n_{0}^{\prime A}=n_{0}^{A}+\left(n_{0}^{Q}-k_{0}\right)$ and $n_{0}^{\prime Q}=k_{0}$. Any such strategy profile is dominated however in terms of conservatives' welfare by the equilibrium of Theorem 3 whenever this exists.
} 
In the equilibrium of Theorem 3 conservatives voting informatively in the originating committee have incentives to do so - whenever relevant - even after observing the vote of the other committee members. These results, however, hold for committee compositions that assure that the ex post bias of conservatives in the receiving committee $\rho_{Q}-\tau_{s_{0}}\left(n_{0}, \sigma_{0}\right)$ is larger than the effective hurdle for conservatives in the receiving committee when liberals vote their bias, $r_{1}-n_{1}^{A}$, so that conservatives in the receiving committee play in equilibrium a k-informative strategy profile with conservative bias.

Can we extend these results when under $\sigma_{0}$ instead $\tau_{s_{0}}\left(n_{0}, \sigma_{0}\right)-\rho_{Q}>n_{1}^{A}-r_{1}$ so that in equilibrium conservatives in the receiving committee play a k-informative strategy profile with liberal bias? First, Lemma 6 shows that for $\theta_{0}+2 \leq t_{0-i}\left(v_{0-i}\right) \leq \bar{\theta}_{0}-2$, $\beta\left(\rho_{Q}+s_{i}\right) \leq \lim _{\alpha \rightarrow 0} \operatorname{Pr}^{(\sigma, \alpha)}\left(\omega_{A} \mid s_{i}, \mathcal{S P}_{i}\left(r_{1}, t_{0-i}\left(v_{0-i}\right)\right)\right) \leq \beta\left(\rho_{Q}+1+s_{i}\right)$, which does not guarantee that a conservative in the originating committee would have incentives to vote informatively after receiving a negative signal conditional on $\underline{\theta}_{0}+2 \leq t_{0-i}\left(v_{0-i}\right) \leq$ $\theta_{0}-2$. To be clear, we introduce the following notion. Suppose that the strategy profile $\sigma^{*}$ is a voting equilibrium given biases $\pi_{Q}, \pi_{A}$. Say that the voting equilibrium $\sigma^{*}$ is generic if it is also a voting equilibrium for any $\beta\left(\rho_{Q}\left(\pi_{Q}\right)-1\right) \leq \pi_{Q}^{\prime} \leq \beta\left(\rho_{Q}\left(\pi_{Q}\right)\right)$ and $\left.\beta\left(\rho_{A}\left(\pi_{A}\right)-1\right)\right) \leq \pi_{A}^{\prime} \leq \beta\left(\rho_{A}\left(\pi_{A}\right)\right)$. Then the conditions in Theorem 3 are necessary and sufficient, in the sense that a voting equilibrium with relevant two-sided informative voting in which all conservatives in the originating committee vote informatively, that is generic and robust to sequential voting within each committee exists if and only if conditions (5) hold (any such voting equilibrium is outcome-equivalent to the equilibrium strategy profile of Theorem 3).

If on the other hand we relax the requirement of a voting equilibria to be robust to sequential voting within each committee, then it is not necessary for the behavior prescribed by $\sigma_{0}$ to be incentive compatible conditional on all feasible $t_{0-i}\left(v_{0-i}\right)$. Instead, it is enough to provide incentives to members of $C_{0}$ in expectation. Theorem 4 then shows that if the number of conservatives and liberals in the originating committee is sufficiently large (so that the inequality $\tau_{s_{0}}\left(t_{0}\left(v_{0}\right), \sigma_{0}\right)-\rho_{Q} \leq\left(\frac{n_{1}-r_{1}}{2}\right)$ holds with equality for a $t_{0}\left(v_{0}\right)$ such that $r_{0}-1 \leq t_{0}\left(v_{0}\right) \leq n_{0}-1$ ), we can (generically) choose $\theta_{0}$ and $\bar{\theta}_{0}$ so that $\sigma_{0}$ is incentive compatible in expectation. In terms of the composition of committees, this requires $n_{0}^{Q}$ to be sufficiently large, and the equivalent to conditions (5) is now

$$
n_{0}^{Q}-\rho_{Q} \geq\left(\frac{n_{1}-1}{2}\right) \text { and } n_{0}^{A} \geq \frac{n_{1}^{Q}-n_{1}^{A}+r_{1}}{2}+\left(r_{0}-\rho_{Q}\right)
$$

Theorem 4 Suppose that $\mathcal{Q}_{1} \in B\left(C_{1}\right)$. If the number of conservatives in $C_{0}$ is sufficiently large, then for any $k_{0} \geq \rho_{Q}$ there exists a voting equilibrium with relevant two-sided informative voting in which $k_{0}$ conservatives in the originating committee vote informatively. Moreover, if conditions (6) hold, there exists a voting equilibrium with relevant two-sided informative voting in which all conservatives in $C_{0}$ vote informatively and

(i) $\mu\left(t_{0} ; \sigma^{*}\right)=0$ for all $r_{0} \leq t_{0}<\underline{\theta}_{0}$.

(ii) $\mu\left(t_{0} ; \sigma^{*}\right) \in(0,1)$ is strictly increasing in $t_{0}$ for all $\underline{\theta}_{0}<t_{0}<\bar{\theta}_{0}$, and

(iii) $\mu\left(t_{0} ; \sigma^{*}\right)=1$ for all $t_{0}>\bar{\theta}_{0}$. 
Proof. The proof is in the appendix.

The previous results in this section assumed that conservatives were a blocking coalition in the receiving committee. Our next result shows that when liberals are a winning coalition in the receiving committee it is necessary and sufficient that liberals also control the originating committee for a voting equilibrium with relevant two sided informative voting that is robust to sequential voting within each committee to exist.

Theorem 5 Assume that $\mathcal{A}_{1} \in W\left(C_{1}\right)$. Then there exists a voting equilibrium with relevant two -sided informative voting that is robust to sequential voting within each committee if and only if $\mathcal{A}_{0} \in W\left(C_{0}\right)$. Moreover, provided that $\frac{n_{0}^{A}-r_{0}^{A}}{2} \leq\left(1-\rho_{A}\right)+r_{1}^{A}$, there exists an equilibrium of this class with $\frac{n_{0}^{A}-r_{0}^{A}}{2}$ informative votes in the originating committee.

Proof. The proof is in the appendix.

\section{Discussion}

In this paper, we develop a simple model of strategic voting with incomplete information and partially common values in sequential multimember committees. The model has stark empirical implications for the analysis of voting in bicameral legislatures, as well as for the interactions between (standing) committees and the floor of legislative bodies and a variety of similar institutional settings in universities and business. It also offers suggestive results for the analysis of sequential electoral systems such as the US presidential election, and sequential referenda such as the one conducted for the ratification of the proposed european constitution. ${ }^{18}$

The model accounts for the basic stylized fact that the outcome of the vote in the first committee typically influences the outcome of the vote in the second committee beyond the binary decision of whether to approve or reject the alternative in the first committee: higher tallies in the first committee are associated with higher success rates of the alternative in the second committee. Having said that, we emphasize the following three main results. First, we show that the receiving committee can act as to modify the effective majority rule for the originating committee, inducing informative voting by some of its members in equilibrium. This is an important feature in settings in which the voting rules do not adjust from issue to issue to the optimal rule à la Austen-Smith and Banks (1996). A key determinant of whether this can in fact occur in equilibrium is the "partisan" composition of the receiving committee, and in particular whether conservatives (biased for the status quo) can block the passage of the proposal in the receiving

\footnotetext{
${ }^{18}$ Iaryczower and Saiegh (2007) (in progress) provides an empirical examination of these hypothesis for the House and Senate of the US Congress.
} 
committee. Second, we show that under some conditions voting equilibria with relevant informative voting in the receiving committee are (Pareto) dominated by equilibria in which all relevant informative voting takes place in the receiving committee, after the alternative is passed unconditionally in the originating committee. Finally, we provide conditions for the existence of an equilibrium with relevant informative voting in both committees, and provide a partial characterization of equilibria of this class. In contrast to voting equilibria with one-sided relevant informative voting in the originating committee, we show that in voting equilibria with relevant two-sided informative voting the conditional probability of the alternative being chosen is strictly increasing in the tally of votes in the originating committee. Moreover, the number of individuals voting informatively in the receiving committee decreases with the difference between the effective majority rule faced by individuals voting informatively in the receiving committee and their effective bias (to evaluate additional information) following a voting outcomes in the originating committee.

We close with two remarks about the model. First, note that while our model assumes that members of the originating and receiving committee receive signals with the same precision, in some circumstances (e.g., committee-floor) it would be desirable to allow for a lower precision of signals of members of the receiving committee. This is, however, a straightforward extension, and all our results continue to hold with minor amendments in this case. A more challenging objection is the possibility of deliberation prior to the vote, which our model ignores completely. We can of course interpret the model as a description of the environment after communication. The question is whether it is plausible to assume that at this point there would still be relevant private information, or whether instead all private information would be transmitted by talking. This will depend on the way we assume players can communicate, and on equilibrium selection. We leave this as an empirical question. If it is, then the vote in the receiving committee should be independent of the outcome of the vote in the originating committee, and behavior in line with the central stylized fact would be due to factors other than the ones considered in this paper. 


\section{Appendix}

Proof of Proposition 2. We first show that $\sigma^{*}$, given by $(i) \sigma_{i}^{*}\left(s_{i}\right)=s_{i}$ for $i \leq k$, and (ii) $\sigma_{i}^{*}\left(s_{i}\right)=-1$ for $i \geq k+1$, is a voting equilibrium if and only if $k=n-(\rho-r)$ and $r \leq \rho \leq R$. Note first that $\tau_{s_{-i}}\left(r-1, \sigma_{-i}^{*}\right)=r-1+n-k$, and therefore $\lambda_{i}^{\mathcal{P}_{i}(C, r)}\left(s_{i}\right)=$ $\beta\left(r-1+n-k+s_{i}\right)$. Incentive compatibility then requires $\lambda_{i}^{\mathcal{P}_{i}(C, r)}(-1)=\beta(r+n-k-2) \leq$ $\pi \leq \beta(r+n-k)=\lambda_{i}^{\mathcal{P}_{i}(C, r)}(1)$. By definition of $\rho$ then either $k=n+(r-\rho)-1$ or $k=n+(r-\rho)$. Proceeding similarly, we can show that the incentive constraint for $i \geq k+1$ implies $k \geq n-(\rho-r)$. Feasibility requires $k \leq n$, and relevant informative voting (that $z=A$ for some $s$ ) that $k \geq R$. With $k=n-(\rho-r)$, these imply that $r \leq \rho \leq R$. It then follows that this strategy profile is a voting equilibrium iff $k=n-(\rho-r)$ and $r \leq \rho \leq R$. Similarly, we can show that $\sigma^{* *}$ such that $(i) \sigma_{i}^{* *}\left(s_{i}\right)=s_{i}$ for $i \leq k$, and (ii) $\sigma_{i}^{* *}\left(s_{i}\right)=1$ for $i \geq k+1$ is a voting equilibrium if and only if $k=n+1-(r-\rho)$ and $-(n-r) \leq \rho \leq r-1$. The result follows, since a voting equilibrium with informative voting must be of one of these classes.

Lemma 1 If $\mathcal{A}_{1} \in W\left(C_{1}\right)$, then for any $k$ such that $\left(1-\rho_{A}\right)+1 \leq k \leq\left(n_{0}^{A}-n_{0}^{Q}\right)-$ $\left(1-\rho_{A}\right)-\left(r_{0}-1\right)$, there exists an EMR voting equilibrium characterized by the pair $\left(k, \theta_{0}(k)\right)=\left(k,\left(n_{0}^{A}-k\right)-n_{0}^{Q}-\left(1-\rho_{A}\right)\right)$ if and only if $\left(1-\rho_{A}\right) \leq \frac{n_{0}^{A}-n_{0}^{Q}-r_{0}}{2}$.

Proof of Lemma 1. First we prove that if $\sigma^{*}$ is a EMR voting equilibrium when $\mathcal{A}_{1} \in W\left(C_{1}\right)$, then it must be the case that conservatives in $C_{0}$ are voting their bias, and liberals are playing a $k$-informative strategy profile with liberal bias. So suppose then that $\sigma^{*}$ is a EMR voting equilibrium. Then there exists an (even) integer $\theta_{0}$, $r_{0}-1 \leq \theta_{0} \leq n_{0}-1$, such that $t_{1}\left(\sigma_{1}^{*}\left(s_{1}, \sigma_{0}^{*}\left(s_{0}\right)\right)\right) \leq r_{1}-1 \forall s_{1}$ whenever $t_{0}\left(\sigma_{0}^{*}\left(s_{0}\right)\right) \leq \theta_{0}-1$. This implies, in particular, that for any $v_{0}=\sigma_{0}^{*}\left(s_{0}\right)$ such that $t_{0}\left(v_{0}\right) \leq \theta_{0}-1$ there is a $i \in A_{1}$ such that $\sigma_{i}^{*}\left(t_{0}\left(v_{0}\right), 1\right)=-1$. Now given that a $R_{1}$-majority is voting against the proposal independently of their signals, then $\lambda_{i}^{\mathcal{P}_{i}\left(C_{1}, r_{1}\right)}\left(s_{i}, v_{0}\right)=\beta\left(\tau_{s_{0}}\left(t_{0}\left(v_{0}\right), \sigma_{0}^{*}\right)+s_{i}\right)$, and therefore incentive compatibility for $i \in A_{1}$ following $v_{0}=\sigma_{0}^{*}\left(s_{0}\right)$ such that $t_{0}\left(v_{0}\right) \leq \theta_{0}-1$ requires $\beta\left(\tau_{s_{0}}\left(t_{0}\left(v_{0}\right), \sigma_{0}^{*}\right)+1\right) \leq \pi_{A}$, or equivalently that $\tau_{s_{0}}\left(\theta_{0}-1, \sigma_{0}^{*}\right) \leq \rho_{A}-2$.

Next we argue that if $i \in Q_{0}$, then $i$ doesn't vote informatively. Suppose to the contrary that for some $i \in Q_{0}, \sigma_{i}\left(\emptyset, s_{i}\right)=s_{i}$. Since in a EMR voting equilibrium $\lambda_{i}^{\mathcal{S P}}\left(s_{i} ; r_{1}\right)=$ $\beta\left(\tau_{s_{0-i}}\left(\theta_{0}, \sigma_{0-i}^{*}\right)+s_{i}\right)$, for incentive compatibility we need either $\tau_{s_{0-i}}\left(\theta_{0} ; \sigma_{0-i}\right)=\rho_{Q}$ or $\tau_{s_{0-i}}\left(\theta_{0} ; \sigma_{0-i}\right)=\rho_{Q}-1$. But then $\tau_{s_{0}}\left(\theta_{0}-1 ; \sigma_{0}\right) \geq \tau_{s_{0-i}}\left(\theta_{0} ; \sigma_{0-i}\right)-1 \geq \rho_{Q}-2>-1 \geq$ $\rho_{A}-2$, which is a contradiction since we have established that $\tau_{s_{0}}\left(t_{0}, \sigma_{0}\right) \leq \rho_{A}-2$ for all $t_{0} \leq \theta_{0}-1$. Then there must exist an $i \in A_{0}$ who is voting informatively. From this it follows that in equilibrium conservatives in $C_{0}$ must vote their bias; i.e., $\sigma_{i}\left(s_{i}\right)=-1$ for all $s_{i} \forall i \in Q_{0} \cdot{ }^{19}$ Moreover, from this it follows that if $i \in A_{0}$ is

${ }^{19}$ Since some liberal is voting informatively, no conservative can be voting informatively as well. Now suppose that at least one conservative in $C_{0}$ is voting for $A$, and let $t_{0}^{N}$ the net tally of conservatives and liberals voting uninformatively in $C_{0}$. Then $\tau_{s_{0}}\left(t_{0}\right)=t_{0}-t_{0}^{N}$. Now suppose $i \in \mathcal{Q}_{0}$ voting for $A$ according to $\sigma$ deviates and votes for $Q$. Conditional on reaching $C_{1}, i$ is taken as a partisan. 
not voting informatively, she must be voting her bias, for otherwise, letting $k$ denote the number of liberals voting informatively, $\tau_{s_{0}}\left(t_{0} ; \sigma_{0}\right)=t_{0}+n_{0}^{Q}+n_{0}^{A}-k$, and thus $\tau_{s_{0}}\left(\theta_{0}-1 ; \sigma_{0}\right) \leq \rho_{A}-2 \Leftrightarrow \theta_{0} \leq k-n_{0}-\left(1-\rho_{A}\right)<r_{0}$. EMR voting equilibria for $\mathcal{A}_{1} \in W\left(C_{1}\right)$ can then be characterized, provided they exist, by pairs $\left(k, \theta_{0}(k)\right)$ such that (i) $\sigma_{i}\left(s, v_{0}\right)=-1(=1) \forall i \in C_{1}, \forall v_{0}=\sigma_{0}\left(s_{0}\right)$ such that $t_{0}\left(v_{0}\right) \leq \theta_{0}-1\left(\geq \theta_{0}+1\right)$, (ii) $\sigma_{i}(s, \emptyset)=-1 \forall i \in \mathcal{Q}_{0}$, and (iii)

$$
\begin{aligned}
& \sigma_{i}(s, \emptyset)=s \quad \forall i \in \mathcal{A}_{0}^{I}(k) \equiv\left\{i \in C_{0}: b_{i}=1, i \leq k\right\} \\
& \sigma_{i}(s, \emptyset)=1 \quad \forall i \in \mathcal{A}_{0}^{N}(k) \equiv\left\{i \in C_{0}: b_{i}=1, i>k\right\}
\end{aligned}
$$

Note then that $t_{0}\left(\sigma\left(s_{0}\right)\right)=-n_{0}^{Q}+\left(n_{0}^{A}-k\right)+\sum_{i \in A_{0}^{I}(k)} s_{i}$, so that $\tau_{s_{0}}\left(t_{0} ; \sigma_{0}\right)=t_{0}+$ $n_{0}^{Q}-\left(n_{0}^{A}-k\right)$, and similarly $\tau_{s_{0-i}}\left(t_{0-i} ; \sigma_{0-i}\right)=t_{0-i}+n_{0}^{Q}-\left(n_{0}^{A}-k\right)$. Since $\lambda_{i}^{\mathcal{S P}}\left(s_{i} ; r_{1}\right)=$ $\beta\left(\tau_{s_{0-i}}\left(\theta_{0}, \sigma_{0-i}^{*}\right)+s_{i}\right)$, for incentive compatibility we need either $\tau_{s_{0-i}}\left(\theta_{0} ; \sigma_{0-i}\right)=\rho_{A}$ or $\tau_{s_{0-i}}\left(\theta_{0} ; \sigma_{0-i}\right)=\rho_{A}-1$. Together with $\tau_{s_{0}}\left(\theta_{0}-1, \sigma_{0}^{*}\right) \leq \rho_{A}-2$, this implies that

$$
\theta_{0}(k)=\left(n_{0}^{A}-k\right)-n_{0}^{Q}-\left(1-\rho_{A}\right)
$$

Since we need $\theta_{0}(k) \geq r_{0}-1$, then $k \leq\left(n_{0}^{A}-n_{0}^{Q}\right)-\left[\left(1-\rho_{A}\right)+\left(r_{0}-1\right)\right]$ (which implies $\left.n_{0}^{A} \geq R_{0}\right)$. Since on the equilibrium path $n_{0}^{A}-n_{0}^{Q}-2 k \leq t_{0} \leq n_{0}^{A}-n_{0}^{Q}$, and thus we need $n_{0}^{A}-n_{0}^{Q}-2 k+1 \leq \theta_{0}(k) \leq n_{0}^{A}-n_{0}^{Q}-1$, then $k \geq 1+\left(1-\rho_{A}\right)$. There exists a $k$ satisfying these two inequalities if and only if $\left(1-\rho_{A}\right) \leq \frac{n_{0}^{A}-n_{0}^{Q}-r_{0}}{2}$. To show that $\sigma^{*}$ is a voting equilibrium for $k: 1+\left(1-\rho_{A}\right) \leq k \leq\left(n_{0}^{A}-n_{0}^{Q}\right)-\left[\left(1-\rho_{A}\right)+\left(r_{0}-1\right)\right]$ and $\theta_{0}(k)=\left(n_{0}^{A}-k\right)-n_{0}^{Q}-\left(1-\rho_{A}\right)$ it only remains to show that members of the originating committee that don't vote informatively do not have profitable deviations. Note that these deviations produce histories that have zero probability. Suppose then that members of the receiving committee treat these deviations as informative: if $i \in \mathcal{A}_{0}^{N}$ votes against the proposal then $j \in C_{1}$ believes $s_{i}=-1$, similarly for $i \in \mathcal{Q}_{0}$. Note that $\sigma_{1}^{*}$ is consistent with these beliefs. Note that if $i \in \mathcal{A}_{0}^{I}$, and $j \in \mathcal{A}_{0}^{N}$, then $\tau_{s_{0-j}}\left(t ; \sigma_{0-j}\right)=\tau_{s_{0-i}}\left(t ; \sigma_{0-i}\right)+1$, and if $\ell \in \mathcal{Q}_{0}$, then $\tau_{s_{0-\ell}}\left(t ; \sigma_{0-j}\right)=\tau_{s_{0-i}}\left(t ; \sigma_{0-i}\right)-1$. It follows from these and the fact that $i \in A_{0}^{I}$ doesn't have a profitable deviation, that no player wants to deviate.

Lemma 2 If $\mathcal{Q}_{1} \in B\left(C_{1}\right)$, there exists a EMR voting equilibrium with $k$ informative votes $\left(1+\rho_{Q} \leq k \leq n_{0}^{Q}\right)$ if and only if $-\left(n_{0}-r_{0}-k\right) \leq 1+\rho_{Q} \leq n_{0}^{Q}$. If all individuals voting uninformatively in $C_{0}$ vote their bias then there exists a EMR voting equilibrium with $k$ informative votes $\left(1+\rho_{Q} \leq k \leq n_{0}^{Q}\right)$ if and only if $n_{0}^{Q} \geq 1+\rho_{Q}$ and $n_{0}^{A} \geq$ $\left(r_{0}-1\right)-\rho_{Q}$.

The deviation therefore only matters if $t_{0-i}=r_{0}-1$ (and it does matter here, since the outcome following $v_{0}$ such that $t_{0-i}=r_{0}$ is $A$ with positive probability). This is a profitable deviation for $i$ iff $\beta\left(\tau_{s_{0-i}}\left(r_{0}-1\right)-1\right)=\beta\left(r_{0}-1-\left(t_{0}^{N}-1\right)-1\right) \leq \beta\left(\rho_{Q}-1\right) \Leftrightarrow r_{0} \leq \rho_{Q}+t_{0}^{N}$. So assume instead that $r_{0}>\rho_{Q}+t_{0}^{N}$. For liberals in $C_{1}$ to vote for $Q$ following $t_{0}=\theta_{0}-1$ we need $\tau_{s_{0}}\left(\theta_{0}-1\right) \leq$ $-\left(1+\left(1-\rho_{A}\right)\right) \Leftrightarrow \theta_{0} \leq t_{0}^{N}-\left(1-\rho_{A}\right)$. For a liberal voting informatively not to have incentives to deviate we need $\beta\left(\tau_{s_{0-i}}\left(\theta_{0}\right)-1\right) \leq \pi_{A} \leq \beta\left(\tau_{s_{0-i}}\left(\theta_{0}\right)+1\right)$, and from this it follows that in fact $\theta_{0}=t_{0}^{N}-\left(1-\rho_{A}\right)$. But since we have assumed that $r_{0}>\rho_{Q}+t_{0}^{N}$, then $r_{0}>\theta_{0}+1$, which is a contradiction with our hypothesis that $\theta_{0} \geq r_{0}-1$. 
Proof of Lemma 2. Proceeding exactly as in the proof of Lemma 1 we can establish that in a EMR voting equilibrium (i) $\tau_{s_{0}}\left(\theta_{0}+1, \sigma_{0}^{*}\right) \geq \rho_{Q}+1$, (ii) $i \in \mathcal{A}_{0}$ doesn't vote informatively, and (iii) there exists $i \in \mathcal{Q}_{0}$ who votes informatively. Equilibrium does not pin down from this the behavior of liberals or conservatives voting uninformatively in $C_{0}$, and as a result for equilibrium purposes we are only concerned in $C_{0}$ with the incentive compatibility constraints of individuals voting informatively. ${ }^{20}$ Now denote the net tally of liberals in $C_{0}$ by $t_{0}^{A}$, and the net tally of conservatives voting uninformatively in $C_{0}$ when $\sigma_{0}$ contains $k$ informative votes by $t_{0}^{Q N}(k)$. Then $\tau_{s_{0}}\left(t_{0}, \sigma_{0}^{*}\right)=t_{0}-t_{0}^{A}-t_{0}^{Q N}(k)$, and therefore (i) above implies $\theta_{0} \geq t_{0}^{A}+t_{0}^{Q N}+\rho_{Q}$. Incentive compatibility of $\sigma$ for $i \in Q_{0}$ requires $\beta\left(\tau_{s_{0-i}}\left(\theta_{0} ; \sigma_{0-i}^{*}\right)-1\right) \leq \pi_{Q} \leq \beta\left(\tau_{s_{0-i}}\left(\theta_{0} ; \sigma_{0-i}^{*}\right)+1\right)$, and therefore either $\tau_{s_{0-i}}\left(\theta_{0} ; \sigma_{0-i}\right)=\rho_{Q}$ or $\tau_{s_{0-i}}\left(\theta_{0} ; \sigma_{0-i}\right)=\rho_{Q}-1$, which together with the previous inequality imply $\theta_{0}=t_{0}^{A}+t_{0}^{Q N}+\rho_{Q}$. For feasibility we need $r_{0}-1 \leq \theta_{0} \leq n_{0}-1$ and $t_{0}^{A}+t_{0}^{Q N}(k)-k+$ $1 \leq \theta_{0} \leq t_{0}^{A}+t_{0}^{Q N}(k)+k-1$, or equivalently $\max \left\{r_{0}-1-t_{0}^{A}-t_{0}^{Q N}(k), 1-k\right\} \leq \rho_{Q} \leq k-1$. This results in two relevant inequalities: $k \geq 1+\rho_{Q}$ (which implies the necessary condition $\left.n_{0}^{Q} \geq 1+\rho_{Q}\right)$ and

$$
\rho_{Q} \geq r_{0}-1-t_{0}^{A}-t_{0}^{Q N}(k)
$$

Note that the right hand side of (8) is minimized when all individuals voting uninformatively in $C_{0}$ vote for $A$, in which case this becomes $\rho_{Q} \geq r_{0}-1-n_{0}+k$, so the maximum number of individuals voting informative is $(k \leq) \rho_{Q}+n_{0}-\left(r_{0}-1\right)$. Note that if instead conservatives in $C_{0}$ voting uninformative vote their bias, (8) becomes $k \geq$ $n_{0}^{Q}-n_{0}^{A}+\left(r_{0}-1\right)-\rho_{Q}$. That is, in this case we need $k \geq \max \left\{1+\rho_{Q}, n_{0}^{Q}-n_{0}^{A}+\left(r_{0}-1\right)-\rho_{Q}\right\}$, and therefore the necessary and sufficient conditions for the existence of a EMR voting equilibrium with any number $1+\rho_{Q} \leq k \leq n_{0}^{Q}$ of conservatives voting informatively and the remaining conservatives voting their bias uninformatively is that $n_{0}^{Q} \geq 1+\rho_{Q}$ and $n_{0}^{A} \geq\left(r_{0}-1\right)-\rho_{Q}$.

Lemma 3 All voting equilibria with relevant informative voting only in the originating committee must be EMR voting equilibria.

Proof of Lemma 3. Suppose not. Then there are at least two cutpoints in the receiving committee. If all of these are below the cutpoint of the EMR voting equilibrium, the IC of members of the originating committee voting informatively would be violated. The same would occur if all the cutpoints are above the cutpoint of the EMR voting equilibrium. Then at least one cutpoint must be below and one above $\theta_{\ell}$. But then the IC of members of the relevant majority in the receiving committee must be violated in some continuation.

To state Lemma 4 formally, we need to develop some terminology. We say that $\sigma$ produces an optimal policy for individuals with bias $b$ given a realization of signals $s$, and

${ }^{20}$ The deviations of individuals voting uninformatively are (when they are supposed to vote against their bias) or can be made to be (when they are supposed to vote for their bias) uninformative, and as a result only matter in the event that $t_{0-i}=r_{0}-1$, but then never, since independently of their vote here the outcome is $Q$ for sure. 
denote this by $O_{b}^{\sigma}(s)=1$, if $t_{0}\left(\sigma_{0}\right) \geq r_{0}$ and $t_{1}\left(\sigma_{1}\left(s_{1}, \sigma_{0}\right)\right) \geq r_{1} \Leftrightarrow t(s) \geq \rho_{b}$. Otherwise we let $O_{b}(s ; \sigma)=0$. That is, we define the mapping $O_{b}(\cdot ; \sigma): S \rightarrow\{0,1\}$ by $O_{b}(s ; \sigma)=1$ if $t_{0}\left(\sigma_{0}\right) \geq r_{0}$ and $t_{1}\left(\sigma_{1}\left(s_{1}, \sigma_{0}\right)\right) \geq r_{1} \Leftrightarrow t(s) \geq \rho_{b}$, and $O_{b}(s ; \sigma)=0$ otherwise. Let $\bar{O}_{b}(\sigma) \equiv \sum_{s: O_{b}(s ; \sigma)=1} \operatorname{Pr}(s)$ denote the probability that $\sigma$ produces an optimal policy for individuals with bias $b$. Given a committee $C^{\prime}$, let $\Sigma_{C^{\prime}}$ denote the set of uninformative and EMR voting equilibria, and let $\bar{\sigma}_{C^{\prime}}$ denote the most informative equilibrium in $\Sigma_{C^{\prime}}$. Let $C(k) \equiv\left\{n_{0}^{Q}(k), n_{0}^{A}(k), n_{1}^{Q}(k), n_{1}^{A}(k)\right\}$. We say that a sequence of committees $\{C(k)\}_{k}$ is increasing if $n_{j}^{b}(k+1)>n_{j}^{b}(k) \forall k, j=0, u$ and $b=Q, A$. We say that a sequence of committees is liberal (conservative) if $\mathcal{A}_{1}(k) \in W\left(C_{1}(k)\right)\left(\mathcal{Q}_{1}(k) \in B\left(C_{1}(k)\right)\right) \forall k$. Then

Lemma 4 (i) For any $\varepsilon>0$ and increasing sequence of conservative committees $\{C(k)\}_{k}$, there exists a $\bar{k}$ such that if $k \geq \bar{k},\left|O_{b}\left(\bar{\sigma}_{C^{k}}\right)-1\right|<\varepsilon$. However, (ii) there exists an $\varepsilon>0$ and an increasing sequence of liberal committees $\{C(k)\}_{k}$ such that $\left|O_{b}\left(\bar{\sigma}_{C^{k}}\right)-1\right|>\varepsilon \forall k$.

Proof of Lemma 4. For (ii), it is enough to note that if along a sequence $n_{0}^{Q}(k) \geq$ $n_{0}^{A}(k)$, the most informative voting equilibrium is the non-informative equilibrium. The result follows, since we can always find an increasing sequence of committees with this property. For (i), note first that there is always a $k^{+}$such that the strategy profile $\sigma^{*}$ in part (i) of Theorem 2 is a voting equilibrium. Therefore for $k \geq k^{+}, \sigma_{C^{\prime}}^{*}$ is the most informative equilibrium in $\Sigma_{C^{\prime}}$. Now $\sum_{i=1}^{n} s_{i} \geq \rho_{Q} \Leftrightarrow \sum_{i=1}^{n} s_{i}^{+} \geq \frac{n+\rho_{Q}}{2} \Leftrightarrow \frac{1}{n} \sum_{i=1}^{n} s_{i}^{+} \geq$ $\frac{1}{2}+\frac{\rho_{Q}}{2 n}$. So suppose that $\omega=\omega_{A}$. Conditional on $\omega_{A}$, signals are i.i.d. Bernoulli $(q)$ random variables. The strong law of large numbers then implies that $\operatorname{Pr}\left(\lim _{n \rightarrow \infty} \frac{1}{n} \sum_{i=1}^{n} s_{i}^{+}=\right.$ $q)=1$, and therefore $($ since $q>1 / 2)$ that $\operatorname{Pr}\left(\lim _{n \rightarrow \infty}\left[\frac{1}{n} \sum_{i=1}^{n} s_{i}^{+}-\left(\frac{1}{2}+\frac{\rho_{Q}}{2 n}\right)\right] \geq 0\right)=1$. However, it also implies that $\operatorname{Pr}\left(\lim _{n_{0}^{Q} \rightarrow \infty}\left[\frac{1}{n_{0}^{Q}} \sum_{i \in Q_{0}} s_{i}^{+}-\left(\frac{1}{2}+\frac{\rho_{Q}}{2 n}\right)\right] \geq 0\right)=1$. Therefore for large committees, conditional on $\omega=\omega_{A}$, both optimality for conservatives and the most informative equilibrium in $\Sigma_{C^{\prime}}$ choose $A$ with probability 1 . A similar argument can be made with $\omega=\omega_{Q}$. This completes the proof.

Proof of Theorem 3. (i) Suppose that liberals in $C_{0}$ vote their bias and that - as hypothesized - conservatives in $C_{0}$ vote informatively. By Proposition 4 , we know that if a MR voting equilibrium $\sigma$ with relevant two-sided informative voting exists, then it must be that there exist $\underline{\theta}_{0}, \theta_{0}$ and $\bar{\theta}_{0}\left(r_{0}-1 \leq \underline{\theta}_{0} \leq \theta_{0} \leq \bar{\theta}_{0} \leq n_{0}+1\right)$, and $\tilde{k}^{0}\left(t_{0}\right), \tilde{k}^{1}\left(t_{0}\right)$ such that liberals in $C_{1}$ vote their bias in any $\Gamma\left(v_{0}\right)$ such that $\underline{\theta}_{0}+1 \leq t_{0}\left(v_{0}\right) \leq \bar{\theta}_{0}-1$ and conservatives in $C_{1}$ play a $\tilde{k}^{0}\left(t_{0}\left(v_{0}\right)\right)$-informative strategy profile with conservative bias for all $v_{0}: \underline{\theta}_{0}+1 \leq t_{0}\left(v_{0}\right) \leq \theta_{0}-1$ and a $\tilde{k}^{1}\left(t_{0}\left(v_{0}\right)\right)$-informative strategy profile with conservative bias for $v_{0}: \theta_{0}+1 \leq t_{0}\left(v_{0}\right) \leq \bar{\theta}_{0}-1$. By Proposition 3 , these strategy profiles are equilibria in $\Gamma\left(v_{0}\right)$ for all $v_{0}: \underline{\theta}_{0}+1 \leq t_{0}\left(v_{0}\right) \leq \theta_{0}-1$ if and only if $\tilde{k}^{0}\left(t_{0}\right)=r_{1}+n_{1}^{Q}-n_{1}^{A}-\rho_{Q}+\left(t_{0}-n_{0}^{A}\right)$ and (from feasibility and relevant informative voting)

$$
\rho_{Q}+n_{0}^{A}-\frac{\left(n_{1}^{Q}-n_{1}^{A}+r_{1}\right)}{2} \leq \underline{\theta}_{0}+1 \leq \theta_{0}-1 \leq n_{1}^{A}+\rho_{Q}+n_{0}^{A}-r_{1}
$$


, and are equilibria in $\Gamma\left(v_{0}\right)$ for all $v_{0}: \theta_{0}+1 \leq t_{0}\left(v_{0}\right) \leq \bar{\theta}_{0}-1$ if and only if $\tilde{k}^{1}\left(t_{0}\right)=$ $\rho_{Q}+n_{1}-\left(r_{1}-1\right)-\left(t_{0}-n_{0}^{A}\right)$ and (from feasibility and relevant informative voting)

$$
n_{1}^{A}+\rho_{Q}+n_{0}^{A}-r_{1}+1 \leq \theta_{0}+1 \leq \bar{\theta}_{0}-1 \leq \rho_{Q}+n_{0}^{A}+\frac{n_{1}-r_{1}}{2}
$$

We want to show that there exist $r_{0}-1 \leq \underline{\theta}_{0} \leq \theta_{0} \leq \bar{\theta}_{0} \leq n_{0}+1$ satisfying (9) and (10) when relevant such that $\beta\left(\rho_{Q}-1+s_{i}\right) \leq \lambda_{i}^{\mathcal{S P}}\left(s_{i} ; r_{1}\right) \leq \beta\left(\rho_{Q}+s_{i}\right)$, eliminating profitable deviations for $i \in \mathcal{Q}_{0}$, where $\lambda_{i}^{\mathcal{S P}}\left(s_{i} ; r_{1}\right)$ is given by (2) with $K(\sigma)=\left\{k: \underline{\theta}_{0} \leq k \leq \bar{\theta}_{0}\right\}$; i.e.,

$$
\lambda_{i}^{\mathcal{S P}}\left(s_{i} ; r_{1}\right)=\sum_{k=\underline{\theta}_{0}}^{\bar{\theta}_{0}} \lim _{\alpha \rightarrow 0} \operatorname{Pr}^{(\sigma, \alpha)}\left(\omega_{A} \mid s_{i}, \mathcal{S} \mathcal{P}_{i}\left(r_{1}, k\right)\right) f\left(s_{i}, k\right)
$$

(ii) Let $\underline{\theta}_{0}=\rho_{Q}+n_{0}^{A}-\frac{\left(n_{1}^{Q}-n_{1}^{A}+r_{1}\right)}{2}-1$, and let $\theta_{0}=\bar{\theta}_{0}=n_{0}+1$. Let $\sigma_{i}\left(v_{0}, s_{i}\right)=-1$ for all $s_{i}$, for all $i \in C_{1}$ for all $v_{0}: t_{0}\left(v_{0}\right) \leq \underline{\theta}_{0}-1$. The conditions $n_{0}^{A} \geq \frac{n_{1}^{Q}-n_{1}^{A}+r_{1}}{2}+\left(r_{0}-\rho_{Q}\right)$ and $n_{0}^{Q}-\rho_{Q} \geq 0$ imply, respectively, that $\underline{\theta}_{0} \geq r_{0}-1$, and $\underline{\theta}_{0} \leq n_{0}-1$. By Lemma $5, \lim _{\alpha \rightarrow 0} \operatorname{Pr}^{(\sigma, \alpha)}\left(\omega_{A} \mid s_{i}, \mathcal{S} \mathcal{P}_{i}\left(r_{1}, \underline{\theta}_{0}\right)=\beta\left(\rho_{Q}-1+s_{i}^{0}\right)\right.$. By Lemma $6, \beta\left(\rho_{Q}-1+s_{i}^{0}\right) \leq$ $\lim _{\alpha \rightarrow 0} \operatorname{Pr}^{(\sigma, \alpha)}\left(\omega_{A} \mid s_{i}, \mathcal{S} \mathcal{P}_{i}\left(r_{1}, \hat{t}_{0}\right)\right) \leq \beta\left(\rho_{Q}+s_{i}^{0}\right)$ for all $v_{0-i}: t_{0-i}\left(v_{0-i}\right)=\hat{t}_{0}$, with $\underline{\theta}_{0}+2 \leq$ $\hat{t}_{0} \leq \theta_{0}-2$. But then we are done, since $n_{0}^{Q}-\rho_{Q} \leq n_{1}^{A}-r_{1}$ implies - noting that $\tilde{k}^{0}\left(\left[n_{1}^{A}+n_{0}^{A}+\rho_{Q}-\left(r_{1}-1\right)\right]-1\right)=n_{1}^{Q}$ - that for all tallies on the equilibrium path $t_{0}\left(\sigma_{0}\left(s_{0}\right)\right.$ (and in fact for all feasible tallies), $t_{0}\left(\sigma_{0}\left(s_{0}\right)\right) \leq \theta_{0}-1$.

Lemma 5 Let $\mathcal{Q}_{1} \in B\left(C_{1}\right)$. Suppose that $\sigma$ is a MR voting equilibrium with relevant two-sided informative voting, and that $i \in C_{0}$ votes informatively. Let $\underline{\theta}_{0}=\rho_{Q}+n_{0}^{A}-$ $\frac{\left(n_{1}^{Q}-n_{1}^{A}+r_{1}\right)}{2}-1$. Then $\lim _{\alpha \rightarrow 0} \operatorname{Pr}^{(\sigma, \alpha)}\left(\omega_{A} \mid s_{i}, \mathcal{S P}_{i}\left(r_{1}, \underline{\theta}_{0}\right)=\beta\left(\rho_{Q}-1+s_{i}^{0}\right)\right.$

Proof of Lemma 5. Fix $v_{0-i}: t_{0-i}\left(v_{0-i}\right)=\underline{\theta}_{0}$. First note that since $t_{0}\left(v_{0-i}^{-}\right)=\theta_{0}-1$, then $t_{1}\left(\sigma_{1}^{*}\left(s_{1}, v_{0-i}^{-}\right)\right) \leq r_{1}-2 \forall s_{1}$, and as a result, $L\left(t_{1}\left(v_{1}^{v_{0-i}^{-}}\right) \leq r_{1}-2, t_{1}\left(v_{1}^{v_{0-i}^{+}}\right) \geq r_{1}\right)=$ $L\left(t_{1}\left(v_{1}^{v_{0-i}^{+}}\right) \geq r_{1}\right)$. Now according to $\sigma^{*}$, this is $t_{1}\left(\sigma_{1}^{*}\left(s_{1}, v_{0-i}^{+}\right)\right)=n_{1}^{A}-\left[n_{1}^{Q}-\tilde{k}^{0}\left(\underline{\theta}_{0}+\right.\right.$ $1)]+\sum_{j=1}^{\tilde{k}^{0}\left(\underline{\theta}_{0}+1\right)} s_{j} \geq r_{1}$, or equivalently, $\sum_{j=1}^{\tilde{k}^{0}\left(\underline{\theta}_{0}+1\right)} s_{j} \geq\left(n_{1}^{Q}-n_{1}^{A}+r_{1}\right)-\tilde{k}^{0}\left(\underline{\theta}_{0}+1\right)$. But $\sum_{j=1}^{\tilde{k}^{0}\left(\underline{\theta}_{0}+1\right)} s_{j} \leq \tilde{k}^{0}\left(\underline{\theta}_{0}+1\right)$. We now choose $\underline{\theta}_{0}+1$ so that $z=A$ following $\underline{\theta}_{0}+1$ is only consistent, according to $\sigma^{*}$, with $\tilde{k}^{0}\left(\underline{\theta}_{0}+1\right)=\frac{\left(n_{1}^{Q}-n_{1}^{A}+r_{1}\right)}{2}$ positive signals:

$$
\underline{\theta}_{0}=\rho_{Q}+n_{0}^{A}-\frac{\left(n_{1}^{Q}-n_{1}^{A}+r_{1}\right)}{2}-1
$$

As a result, $t_{1}\left(\sigma_{1}^{*}\left(s_{1}, v_{0-i}^{+}\right)\right) \geq r_{1} \Leftrightarrow s_{1} \in\left\{s_{1}: t_{1}\left(s_{1}\right)=\left(\frac{n_{1}^{Q}-n_{1}^{A}+r_{1}}{2}\right)\right\}$, and $L\left(t_{1}\left(v_{1}^{v_{0-i}^{+}}\right) \geq\right.$ $1)=\left(\frac{1-q}{q}\right)^{\left(\frac{n_{1}^{Q}-n_{1}^{A}+r_{1}}{2}\right)}$. Note next that $\tau_{s_{0-i}}\left(t, \sigma_{0-i}^{*}\right)=t-n_{0}^{A}$, so that $\tau_{s_{0-i}}\left(\underline{\theta}_{0}, \sigma_{0-i}^{*}\right)=$ 
$\rho_{Q}-\left(\frac{n_{1}^{Q}-n_{1}^{A}+r_{1}}{2}\right)-1$, and therefore $L\left(v_{0-i}: t_{0-i}\left(v_{0-i}\right)=\underline{\theta}_{0}\right)=\left(\frac{1-q}{q}\right)^{\rho_{Q}-\left(\frac{n_{1}^{Q}-n_{1}^{A}+1}{2}\right)-1}$. Putting these observations together we obtain the result.

Lemma 6 Let $\mathcal{Q}_{1} \in B\left(C_{1}\right)$. Suppose that $\sigma$ is a $M R$ voting equilibrium with relevant two-sided informative voting, and that $i \in C_{0}$ votes informatively.

(i) Let $v_{0-i}$ be a voting outcome in $C_{0-i}$ such that $\underline{\theta}_{0}+2 \leq t_{0-i}\left(v_{0-i}\right) \leq \theta_{0}-2$. Then

$$
\beta\left(\rho_{Q}-1+s_{i}\right) \leq \lim _{\alpha \rightarrow 0} \operatorname{Pr}^{(\sigma, \alpha)}\left(\omega_{A} \mid s_{i}, \mathcal{S P}_{i}\left(r_{1}, t_{0-i}\left(v_{0-i}\right)\right)\right) \leq \beta\left(\rho_{Q}+s_{i}\right)
$$

(ii) Let $v_{0-i}$ be a voting outcome in $C_{0-i}$ such that $\theta_{0}+2 \leq t_{0-i}\left(v_{0-i}\right) \leq \bar{\theta}_{0}-2$. Then

$$
\beta\left(\rho_{Q}+s_{i}\right) \leq \lim _{\alpha \rightarrow 0} \operatorname{Pr}^{(\sigma, \alpha)}\left(\omega_{A} \mid s_{i}, \mathcal{S P}_{i}\left(r_{1}, t_{0-i}\left(v_{0-i}\right)\right)\right) \leq \beta\left(\rho_{Q}+1+s_{i}\right)
$$

Proof of Lemma 6. Consider part (i). Fix $v_{0-i}: t_{0-i}\left(v_{0-i}\right)=\hat{t}_{0}$ for $\underline{\theta}_{0}+2 \leq \hat{t}_{0} \leq$ $\theta_{0}-2$. We know already that $\tau_{s_{0-i}}\left(t, \sigma_{0-i}^{*}\right)=t-n_{0}^{A}$, so that $L\left(v_{0-i}: t_{0-i}\left(v_{0-i}\right)=\hat{t}_{0}\right)=$ $\left(\frac{1-q}{q}\right)^{\hat{t}_{0}-n_{0}^{A}}$. Next note that according to $\sigma^{*}, i$ 's vote is influential only if $t_{1}\left(\sigma_{1}^{*}\left(s_{1}, v_{0-i}^{-}\right)\right) \leq$ $r-2$ and $t_{1}\left(\sigma_{1}^{*}\left(s_{1}, v_{0-i}^{+}\right)\right) \geq r$. But $t_{1}\left(\sigma_{1}^{*}\left(s_{1}, v_{0-i}^{-}\right)\right)=n_{1}^{A}-\left(n_{1}^{Q}-\tilde{k}^{0}\left(\hat{t}_{0}-1\right)\right)+\sum_{i=1}^{\tilde{k}^{0}\left(\hat{t}_{0}-1\right)} s_{i} \leq$ $r-2$, or if and only if for some labeling of the individuals voting informatively

$$
\sum_{i=1}^{\tilde{k}^{0}\left(\hat{t}_{0}-1\right)} s_{i} \leq \rho_{Q}+n_{0}^{A}-\hat{t}_{0}-1
$$

Similarly $t_{1}\left(\sigma_{1}^{*}\left(s_{1}, v_{0-i}^{+}\right)\right) \geq r$ if and only if

$$
\sum_{i=1}^{\tilde{k}^{0}\left(\hat{t}_{0}+1\right)} s_{i}=\sum_{i=1}^{\tilde{k}^{0}\left(\hat{t}_{0}-1\right)} s_{i}+\sum_{i=\tilde{k}^{0}\left(\hat{t}_{0}-1\right)+1}^{\tilde{k}^{0}\left(\hat{t}_{0}+1\right)} s_{i} \geq \rho_{Q}+n_{0}^{A}-\hat{t}_{0}-1
$$

Since $\sum_{i=\tilde{k}^{0}\left(\hat{t}_{0}-1\right)+1}^{\tilde{k}^{0}\left(\hat{t}_{0}+1\right)} s_{i} \leq 2$, this implies that to the knowledge that $\sum_{i=1}^{n_{0}^{Q}} s_{i}=\hat{t}_{0}-n_{0}^{A}$ in $C_{0}$, we must add $\sum_{i=1}^{\tilde{k}^{0}\left(\hat{t}_{0}-1\right)-1} s_{i}=\rho_{Q}+n_{0}^{A}-\hat{t}_{0}-2, s_{\tilde{k}^{0}\left(\hat{t}_{0}+1\right)}=1$ and $\left(s_{\tilde{k}^{0}\left(\hat{t}_{0}-1\right)}, s_{\tilde{k}^{0}\left(\hat{t}_{0}-1\right)+1}\right) \in$ $\{(-1,1) ;(1,-1) ;(1,1)\}$ (or permutations thereof). Denoting this latter event by $Z$, we have $\frac{\operatorname{Pr}\left(Z \mid \omega_{Q}\right)}{\operatorname{Pr}\left(Z \mid \omega_{A}\right)}=\frac{2 q(1-q)+(1-q)^{2}}{2 q(1-q)+q^{2}}>\frac{1-q}{q}$, and therefore $\frac{\operatorname{Pr}\left(Z \mid \omega_{Q}\right)}{\operatorname{Pr}\left(Z \mid \omega_{A}\right)}=\mu \frac{1-q}{q}$ for some $\mu>1$. Thus

$$
\lim _{\alpha \rightarrow 0} \operatorname{Pr}^{(\sigma, \alpha)}\left(\omega_{A} \mid s_{i}, \mathcal{S P}_{i}\left(r_{1}, \hat{t}_{0}\right)\right)=\frac{1}{1+\mu\left(\frac{1-q}{q}\right)^{\rho_{Q}+s_{i}}}=\frac{1}{1+\left(\frac{1-q}{q}\right)^{\rho_{Q}+s_{i}-x}}
$$


for some $x \in(0,1)$. Equivalently, $\lim _{\alpha \rightarrow 0} \operatorname{Pr}^{(\sigma, \alpha)}\left(\omega_{A} \mid s_{i}, \mathcal{S P}_{i}\left(r_{1}, \hat{t}_{0}\right)\right)=\beta\left(\rho_{Q}+s_{i}-x\right)$ for some $x \in(0,1)$, which implies the claim. Part (ii) follows from the same argument, noting that inequalities (13) and (14) become $\sum_{i=1}^{\tilde{k}^{1}\left(\hat{t}_{0}-1\right)} s_{i} \leq \rho_{Q}+n_{0}^{A}-\hat{t}_{0}$ and $\sum_{i=1}^{\tilde{k}^{1}\left(\hat{t}_{0}+1\right)} s_{i} \geq$ $\rho_{Q}+n_{0}^{A}-\hat{t}_{0}$.

Proof of Theorem 4. Repeat here step (i) in the proof of Proposition 3. Next let $\sigma_{i}\left(v_{0}, s_{i}\right)=-1(=1)$ for all $s_{i}$, for all $i \in C_{1}$ for all $v_{0}: t_{0}\left(v_{0}\right) \leq \underline{\theta}_{0}-1\left(\geq \bar{\theta}_{0}+1\right)$. Let $\underline{\theta}_{0}=\rho_{Q}+n_{0}^{A}-\frac{\left(n_{1}^{Q}-n_{1}^{A}+r_{1}\right)}{2}-1$. As before, the conditions $n_{0}^{A} \geq \frac{n_{1}^{Q}-n_{1}^{A}+r_{1}}{2}+\left(r_{0}-\rho_{Q}\right)$ and $n_{0}^{Q}-\rho_{Q} \geq 0$ imply, respectively, that $\underline{\theta}_{0} \geq r_{0}-1$, and $\underline{\theta}_{0} \leq n_{0}-1$. By Lemma 5, $\lim _{\alpha \rightarrow 0} \operatorname{Pr}^{(\sigma, \alpha)}\left(\omega_{A} \mid s_{i}, \mathcal{S} \mathcal{P}_{i}\left(r_{1}, \underline{\theta}_{0}\right)=\beta\left(\rho_{Q}-1+s_{i}^{0}\right)\right.$. Now if $n_{0}^{Q}-\rho_{Q} \leq n_{1}^{A}-r_{1}$, we know that the result holds by Proposition 3. Therefore assume to the contrary that $n_{1}^{A}-r_{1}+2 \leq n_{0}^{Q}-\rho_{Q}$, so that $\max _{s_{0}} t_{0}\left(\sigma_{0}\left(s_{0}\right)\right) \geq \theta_{0}+1$ for all $\theta_{0}$ satisfying $(9)$. Let $\theta_{0}=n_{1}^{A}+n_{0}^{A}+\rho_{Q}-\left(r_{1}-1\right)$. By Lemma 6, $\beta\left(\rho_{Q}-1+s_{i}^{0}\right) \leq \lim _{\alpha \rightarrow 0} \operatorname{Pr}^{(\sigma, \alpha)}\left(\omega_{A} \mid s_{i}, \mathcal{S} \mathcal{P}_{i}\left(r_{1}, \hat{t}_{0}\right)\right) \leq \beta\left(\rho_{Q}+s_{i}^{0}\right)$ for all $v_{0-i}$ : $t_{0-i}\left(v_{0-i}\right)=\hat{t}_{0}$, with $\underline{\theta}_{0}+2 \leq \hat{t}_{0} \leq \theta_{0}-2$. We now show that there exists a $\bar{\theta}_{0} \geq \theta_{0}$ satisfying (10) such that $\beta\left(\rho_{Q}-1+s_{i}\right) \leq \lambda_{i}^{\mathcal{S P}}\left(s_{i} ; r_{1}\right) \leq \beta\left(\rho_{Q}+s_{i}\right)$, eliminating deviations for $i \in \mathcal{Q}_{0}$. In what follows we will make explicit the dependency of $\lambda_{i}^{\mathcal{S P}}\left(s_{i} ; r_{1}\right)$ on $\bar{\theta}_{0}$ in $\sigma^{*}$ by writing $\lambda_{i}^{\mathcal{S P}}\left(s_{i} ; r_{1}, \bar{\theta}_{0}\right)$ (here we fix $\underline{\theta}_{0}$ and $\theta_{0}$ at the values specified above).

First note that if $\bar{\theta}_{0}=\theta_{0}=n_{1}^{A}+n_{0}^{A}+\rho_{Q}-\left(r_{1}-1\right)$, then $\lambda_{i}^{\mathcal{S P}}\left(s_{i} ; r_{1} ; \bar{\theta}_{0}\right) \leq \beta\left(\rho_{Q}+s_{i}\right)$. To see this note that since $Q$ loses against $A$ independently of $s_{1}$ in $\Gamma\left(v_{0-i}^{+}\right), L\left(\mathcal{S P}_{i}\left(r_{1}, \bar{\theta}_{0}\right)\right)=$ $L\left(t_{1}\left(v_{1}^{v_{0-i}^{-}}\right) \leq r_{1}-2\right)=L\left(\sum_{j=1}^{n_{1}^{Q}} s_{j} \leq r_{1}-2-n_{1}^{A}\right) \geq\left(\frac{1-q}{q}\right)^{\left(r_{1}-2-n_{1}^{A}\right)}$ by Lemma 7 . Since also $\tau_{s_{0-i}}\left(\bar{\theta}_{0}, \sigma_{0-i}^{*}\right)=n_{1}^{A}+\rho_{Q}-\left(r_{1}-1\right)$, and thus $L\left(v_{0-i}: t_{0-i}\left(v_{0-i}\right)=\bar{\theta}_{0}\right)=$ $\left(\frac{1-q}{q}\right)^{n_{1}^{A}+\rho_{Q}-\left(r_{1}-1\right)}$, we have $\lim _{\alpha \rightarrow 0} \operatorname{Pr}^{(\sigma, \alpha)}\left(\omega_{A} \mid s_{i}, \mathcal{S} \mathcal{P}_{i}\left(r_{1}, \hat{t}_{0}\right)\right) \leq \beta\left(\rho_{Q}+s_{i}-1\right)$. If also $\lambda_{i}^{\mathcal{S P}}\left(s_{i} ; r_{1} ; \bar{\theta}_{0}\right) \geq \beta\left(\rho_{Q}+s_{i}-1\right)$, then we are done. So suppose not. Then in equilibrium $\bar{\theta}_{0} \geq \theta_{0}+2$. Since $\tilde{k}^{0}\left(\theta_{0}-1\right)=n_{1}^{Q}$ and $\tilde{k}^{1}\left(\theta_{0}+1\right)=n_{1}^{Q}-1, t_{1}\left(\sigma_{1}^{*}\left(s_{1}, v_{0-i}^{-}\right)\right) \leq r_{1}-2$ and $t_{1}\left(\sigma_{1}^{*}\left(s_{1}, v_{0-i}^{+}\right)\right) \geq r_{1}$ only if $\sum_{i=1}^{n_{1}^{Q}} s_{i}=r_{1}-2-n_{1}^{A}$. Then $L\left(\mathcal{S P}_{i}\left(r_{1}, \theta_{0}\right)\right)=\left(\frac{1-q}{q}\right)^{r_{1}-2-n_{1}^{A}}$, which with $L\left(v_{0-i}: t_{0-i}\left(v_{0-i}\right)=\theta_{0}\right)=\left(\frac{1-q}{q}\right)^{n_{1}^{A}+\rho_{Q}-r_{1}+1}$ gives $\lim _{\alpha \rightarrow 0} \operatorname{Pr}^{(\sigma, \alpha)}\left(\omega_{A} \mid s_{i}, \mathcal{S} \mathcal{P}_{i}\left(r_{1}, \theta_{0}\right)\right)=$ $\beta\left(\rho_{Q}-1+s_{i}^{0}\right)$. Now, by Lemma 6 , (a) for all $v_{0-i}: t_{0-i}\left(v_{0-i}\right)=\hat{t}_{0}$ such that $\theta_{0}+2 \leq$ $\hat{t}_{0} \leq \bar{\theta}_{0}-2$, we have $\beta\left(\rho_{Q}+s_{i}^{0}\right) \leq \lim _{\alpha \rightarrow 0} \operatorname{Pr}^{(\sigma, \alpha)}\left(\omega_{A} \mid s_{i}, \mathcal{S P}_{i}\left(r_{1}, \hat{t}_{0}\right)\right) \leq \beta\left(\rho_{Q}+1+s_{i}^{0}\right)$, but (b) for all $v_{0-i}: t_{0-i}\left(v_{0-i}\right)=\hat{t}_{0}$ such that $\underline{\theta}_{0}+2 \leq \hat{t}_{0} \leq \theta_{0}-2$ instead $\beta\left(\rho_{Q}-1+s_{i}^{0}\right) \leq$ $\lim _{\alpha \rightarrow 0} \operatorname{Pr}^{(\sigma, \alpha)}\left(\omega_{A} \mid s_{i}, \mathcal{S P}_{i}\left(r_{1}, \hat{t}_{0}\right)\right) \leq \beta\left(\rho_{Q}+s_{i}^{0}\right)$. Together with the fact that (by Lemma 7) $\lim _{\alpha \rightarrow 0} \operatorname{Pr}^{(\sigma, \alpha)}\left(\omega_{A} \mid s_{i}, \mathcal{S P}_{i}\left(r_{1}, \bar{\theta}_{0}\right)\right)$ is also increasing in $\bar{\theta}_{0}$, this implies that $\lambda_{i}^{\mathcal{S P}}\left(s_{i} ; r_{1} ; \bar{\theta}_{0}\right)$ is strictly increasing in $\bar{\theta}_{0}$ for $\bar{\theta}_{0} \geq \theta_{0}+2$. Finally, note that if we choose

$$
\bar{\theta}_{0}=\rho_{Q}+n_{0}^{A}+1+\left(\frac{n_{1}-r_{1}}{2}\right)
$$

and this is feasible, in the sense that $\bar{\theta}_{0}=\rho_{Q}+n_{0}^{A}+\left(\frac{n_{1}+1}{2}\right) \leq n_{0}+1$ (which is assumed true in the hypothesis $)$, then $\lim _{\alpha \rightarrow 0} \operatorname{Pr}^{(\sigma, \alpha)}\left(\omega_{A} \mid s_{i}, \mathcal{S} \mathcal{P}_{i}\left(r_{1}, \bar{\theta}_{0}\right)\right)=\beta\left(\rho_{Q}+s_{i}\right)$. This follows 
since $L\left(\mathcal{S P}_{i}\left(r_{1}, \bar{\theta}_{0}\right)\right)=L\left(t_{1}\left(v_{1}^{v_{0-i}^{-}}\right) \leq r_{1}-2\right)$, and according to $\sigma^{*}, t_{1}\left(\sigma_{1}^{*}\left(s_{1}, v_{0-i}^{-}\right)\right)=$ $n_{1}^{A}+\left[n_{1}^{Q}-\tilde{k}^{1}\left(\bar{\theta}_{0}-1\right)\right]+\sum_{j=1}^{\tilde{k}^{1}\left(\bar{\theta}_{0}-1\right)} s_{j} \leq r_{1}-2 \Leftrightarrow \sum_{j=1}^{\tilde{k}^{1}\left(\bar{\theta}_{0}-1\right)} s_{j} \leq \rho_{Q}+n_{0}^{A}-\bar{\theta}_{0}$, but $\sum_{j=1}^{\tilde{k}^{1}\left(\bar{\theta}_{0}-1\right)} s_{j} \geq-\tilde{k}^{1}\left(\bar{\theta}_{0}-1\right)=-\rho_{Q}-n_{1}-n_{0}^{A}+\bar{\theta}_{0}+r_{1}-2$. Therefore with $\bar{\theta}_{0}$ as in (15), we have $L\left(\mathcal{S P}_{i}\left(r_{1}, \bar{\theta}_{0}\right)\right)=\left(\frac{1-q}{q}\right)^{\rho_{Q}+n_{0}^{A}-\bar{\theta}_{0}}$, and hence $\lim _{\alpha \rightarrow 0} \operatorname{Pr}(\sigma, \alpha)\left(\omega_{A} \mid s_{i}, \mathcal{S P}_{i}\left(r_{1}, \bar{\theta}_{0}\right)\right)=$ $\beta\left(\rho_{Q}+s_{i}\right)$. Therefore $\beta\left(\rho_{Q}-1+s_{i}\right) \leq \lambda_{i}^{\mathcal{S P}}\left(s_{i} ; r_{1} ; \bar{\theta}_{0}\right) \leq \beta\left(\rho_{Q}+1+s_{i}\right)$. But then there exists a $\bar{\theta}_{0}: n_{1}^{A}+n_{0}^{A}+\rho_{Q}-\left(r_{1}-1\right) \leq \bar{\theta}_{0} \leq \rho_{Q}+n_{0}^{A}+\left(\frac{n_{1}+1}{2}\right)$ such that $\beta\left(\rho_{Q}-1+s_{i}\right) \leq$ $\lambda_{i}^{\mathcal{S P}}\left(s_{i} ; r_{1} ; \bar{\theta}_{0}\right) \leq \beta\left(\rho_{Q}+s_{i}\right)$.

Lemma 7 (i) $\operatorname{Pr}\left(\omega_{A} \mid \sum_{i=1}^{k} s_{i} \leq k-A\right)$ is decreasing in $k$ and $A$, and (ii) $\operatorname{Pr}\left(\omega_{A} \mid \sum_{i=1}^{k} s_{i} \leq\right.$ $k-A)<\operatorname{Pr}\left(\omega_{A} \mid \sum_{i=1}^{k} s_{i}=k-A\right)$

Proof of Lemma 7. For $(i)$, it is enough to show that $\frac{\operatorname{Pr}\left(\sum_{i=1}^{k} s_{i} \leq k-A \mid \omega_{Q}\right)}{\operatorname{Pr}\left(\sum_{i=1}^{k} s_{i} \leq k-A \mid \omega_{A}\right)}$ is increasing in $k$ and $A$. But this follows since

$$
\frac{\operatorname{Pr}\left(\sum_{i=1}^{k} s_{i} \leq k-A \mid \omega_{Q}\right)}{\operatorname{Pr}\left(\sum_{i=1}^{k} s_{i} \leq k-A \mid \omega_{A}\right)}=\frac{\operatorname{Pr}\left(\left|s^{-}\right| \geq \frac{A}{2} \mid \omega_{Q}\right)}{\operatorname{Pr}\left(\left|s^{-}\right| \geq \frac{A}{2} \mid \omega_{A}\right)}=\frac{\operatorname{Pr}\left(\left|s^{+}\right| \geq \frac{A}{2} \mid \omega_{A}\right)}{\operatorname{Pr}\left(\left|s^{+}\right| \geq \frac{A}{2} \mid \omega_{Q}\right)}=\frac{\sum_{t=A / 2}^{k} F\left(t ; \omega_{A}\right)}{\sum_{t=A / 2}^{k} F\left(t ; \omega_{Q}\right)}
$$

where $F\left(t ; \omega_{A}\right) \equiv\left(\begin{array}{c}k \\ t\end{array}\right) q^{t}(1-q)^{k-t}$ and $F\left(t ; \omega_{Q}\right) \equiv\left(\begin{array}{c}k \\ t\end{array}\right)(1-q)^{t} q^{k-t}$, and $\frac{F\left(k+1 ; \omega_{A}\right) / F\left(k+1 ; \omega_{Q}\right)}{F\left(k ; \omega_{A}\right) / F\left(k ; \omega_{Q}\right)}=\left(\frac{q}{1-q}\right)^{2}>1$, so that $\frac{F\left(k+1 ; \omega_{A}\right)}{F\left(k+1 ; \omega_{Q}\right)}>\frac{F\left(k ; \omega_{A}\right)}{F\left(k ; \omega_{Q}\right)}$. This also implies (ii).

Proof of Theorem 5. We sketch the argument, the details can be filled using the steps in previous results. First, proceeding as in Lemma 6, we can show that if $\mathcal{A}_{1} \in W\left(C_{1}\right), \sigma$ is a MR voting equilibrium with relevant two-sided informative voting, and $i \in C_{0}$ votes informatively, then for any $v_{0-i}$ such that $\underline{\theta}_{0}+2 \leq t_{0-i}\left(v_{0-i}\right) \leq \theta_{0}-2$.

$$
\beta\left(\rho_{A}-2+s_{i}\right) \leq \lim _{\alpha \rightarrow 0} \operatorname{Pr}^{(\sigma, \alpha)}\left(\omega_{A} \mid s_{i}, \mathcal{S P}_{i}\left(r_{1}, t_{0-i}\left(v_{0-i}\right)\right)\right) \leq \beta\left(\rho_{A}-1+s_{i}\right)
$$

, while for any $v_{0-i}$ such that $\theta_{0}+2 \leq t_{0-i}\left(v_{0-i}\right) \leq \bar{\theta}_{0}-2$

$$
\beta\left(\rho_{A}-1+s_{i}\right) \leq \lim _{\alpha \rightarrow 0} \operatorname{Pr}^{(\sigma, \alpha)}\left(\omega_{A} \mid s_{i}, \mathcal{S P}_{i}\left(r_{1}, t_{0-i}\left(v_{0-i}\right)\right)\right) \leq \beta\left(\rho_{A}+s_{i}\right)
$$

From this it follows immediately that if $\sigma$ is robust to sequential voting within each committee, then only liberals in $C_{0}$ can vote informatively. But then (by the same argument as in footnote 19) conservatives in $C_{0}$ must be voting their bias, and hence if $\mathcal{Q}_{0} \in C_{0}$ there can't be relevant informative in $C_{0}$. Next suppose that in equilibrium conservatives in the originating committee vote their bias, and liberals play a $k$-informative strategy profile with liberal bias. Note that if $k \leq \frac{n_{0}^{A}-\left(n_{0}^{Q}+r_{0}\right)}{2}$, then $t_{0}\left(\sigma_{0}\right)=-n_{0}^{Q}+\left(n_{0}^{A}-k\right)+\sum_{i=1}^{k} s_{i} \geq r_{0}$ for all $s_{0}$. So assume in fact that $1 \leq k \leq \frac{n_{0}^{A}-\left(n_{0}^{Q}+r_{0}\right)}{2}$ (this is possible since $\mathcal{A}_{0} \in W\left(C_{0}\right)$ ). 
Then $\tau_{s_{0}}\left(t_{0}, \sigma_{0}\right) \geq-k \geq-\frac{n_{0}^{A}-\left(n_{0}^{Q}+r_{0}\right)}{2}$ for any $s_{0}$, and thus by the assumption in the hypothesis $\left(1-\rho_{A}\right)+\tau_{s_{0}}\left(t_{0}, \sigma_{0}\right) \geq-\left(n_{1}^{Q}+r_{1}\right)$ for any $s_{0}$ (note that for $k=1$ this condition is always satisfied). But this, together with Proposition 3, implies that if there is relevant informative voting in $\Gamma\left(v_{0}\right)$ then liberals play a k-strategy profile with liberal bias, or equivalently that for any such $v_{0-i}=\sigma_{0-i}\left(s_{0-i}\right), \theta_{0}+2 \leq t_{0-i}\left(v_{0-i}\right) \leq \bar{\theta}_{0}-2$ (i.e., that in equilibrium $\left.\underline{\theta}_{0}=\theta_{0}\right)$. If $\tau_{s_{0}}\left(t_{0}, \sigma_{0}\right)+1-\rho_{A}<\frac{n_{1}^{A}-n_{1}^{Q}-r_{1}}{2}$, then we are done. Otherwise, set $\bar{\theta}_{0}$ so that $\tau_{s_{0}}\left(\bar{\theta}_{0}-1, \sigma_{0}\right)+1-\rho_{A}=\frac{n_{1}^{A}-n_{1}^{Q}-r_{1}}{2}$. 


\section{References}

Ali, N. S., And N. Kartik (2006): "A Theory of Momentum in Sequential Voting," Department of Economics, UCSD.

Austen-Smith, D., And J. S. Banks (1996): "Information Aggregation, Rationality, and the Condorcet Jury Theorem," American Political Science Review, 90, 34-45.

Austen-Smith, D., And J. Duggan (eds.) (2005): Social Choice and Strategic Decisions: Essays in Honor of Jeffrey S. Banks.

Austen-Smith, D., And T. Feddersen (2005): "Deliberation and Voting Rules," in Austen-Smith and Duggan (2005), pp. 269-316.

(2006): "Deliberation, Preference Uncertainty, and Voting Rules," American Political Science Review, 100, 209-218.

Battaglini, M. (2005): "Sequential Voting with Abstention," Games and Economic Behavior, 51, 445-463.

Battaglini, M., R. Morton, and T. Palfrey (2007): "Efficiency, Equity and Timing of Voting Mechanisms," Forthcoming, American Political Science Review.

Callender, S. (2007): "Bandwagons and Momentum in Sequential Voting," Forthcoming, Review of Economic Studies.

Coughlan, P. (2000): "In Defense of Unanimous Jury Veredicts: Mistrials, Communication, and Strategic Voting," American Political Science Review, 94, 375-393.

Crawford, V. P., And J. Sobel (1982): "Strategic Information Transmission," Econometrica, 50, 1431-1451.

Cutrone, M., and N. McCarty (2005): "Does Bicameralism Matter?," Unpublished Article. Princeton University.

Dekel, E., And M. Piccione (2000): "Sequential Voting Procedures in Symmetric Binary Elections," Journal of Political Economy, 108, 34-54.

Feddersen, T., And W. Pesendorfer (1997): "Voting Behavior and Information Aggregation in Elections With Private Information," Econometrica, 65, 1029-1058.

(1998): "Convicting the Innocent: The Inferiority of Unanimous Jury Verdicts under Strategic Voting," American Political Science Review, 92, 23-35.

FEy, M. (1998): "Informational Cascades and Sequential Voting," Working Paper, University of Rochester.

Gerardi, D., And L. YARIV (2007): "Deliberative Voting," Journal of Economic Theory, 134, 317-338. 
Gilligan, T., And K. Krehbiel (1987): "Collective Decision-Making and Standing Committees: An Informational Rationale for Restrictive Amendment Procedures," Journal of Law, Economics and Organization, 3, 287-335.

IARYCZOWER, M. (2007): "Strategic Voting in Sequential Committees, Part II: Efficiency," HSS, California Institute of Technology.

IARYCZOWER, M., AND S. SAIEGH (2007): "An Empirical Examination of Strategic Voting and Information Aggregation in Bicameral Legislatures: The US Congress," HSS, California Institute of Technology.

Maug, E., And B. Yilmaz (2002): "Two Class Voting: A Mechanism for Conflict Resolution," American Economic Review, 92, 1448-1471.

Oleszek, W. J. (2004): Congressional Procedures and the Policy Process. Congressional Quarterly Inc., Washington, D.C.

Persico, N. (2004): "Committee Design with Endogenous Information," Review of Economic Studies, 71, 165-191.

Piketty, T. (2000): "Voting as Communicating," Review of Economic Studies, 67, 169-191.

RAZIN, R. (2003): "Signaling and Election Motivations in a Voting Model with Common Values and Responsive Candidates," Econometrica, 71, 1083-1119.

Tsebelis, G., And J. Money (1997): Bicameralism. Cambridge University Press, Cambridge, UK.

WiT, J. (1997): "Herding Behavior in a Roll-Call Voting Game," Unpublished Article, University of Amsterdam. 